\title{
The quantity, quality and characteristics of Aboriginal and Torres Strait Islander Australian mentoring literature: a systematic review
}

\author{
Roxanne Bainbridge ${ }^{1 *}$, Komla Tsey $^{1}$, Janya McCalman ${ }^{1}$ and Simon Towle ${ }^{2}$
}

\begin{abstract}
Background: Mentoring is a key predictor of empowerment and prospectively a game changer in the quest to improve health inequities. This systematic review reports on the state of evidence on mentoring for Indigenous Australians by identifying the quantity, nature, quality and characteristics of mentoring publications.
\end{abstract}

Methods: Thirteen databases were searched using specific search strings from 1983 - 2012. Grey literature was also canvassed. The resultant publications were mined to identify their outputs, nature, and quality. These were then conceptually mined for their characteristics to develop a model of mentoring that included the initiating environments, facilitating environments, operational strategies and outcomes.

Results: 771 citations were identified; 37 full text publications met inclusion criteria and were assessed. Fifteen were eligible for review. Four of five original research publications used strong qualitative research designs. No publications were found before 1999; the largest proportion concentrated in $2011(n=4)$. Facilitating environments included: mapping participants' socio-cultural and economic context; formal mentoring practices with internal flexibility; voluntary participation; integrated models with wrap-around services; mentor/staff competencies; and sustained funding. Mentoring strategies comprised: holistic scaffolding approaches; respectful, trusting, one-on-one mentoring relationships; knowledgeable mentors; regular contact; longer-term relationships and exit strategies; culturally-tailored programs; personal and social development opportunities; and specialised skills and learning opportunities. Outcomes varied in accordance to program aims and included improvements in aspects of education and employment, offending behaviours, relationships, and personal, social and professional development.

Conclusion: Little research explored the effectiveness of mentoring, captured its impact qualitatively or quantitatively, developed appropriate measures or assessed its cost-effectiveness. There is a real need to evaluate programs particularly in terms of outcomes and, given there were no economic evaluations, costs. Commitments to improving Indigenous Australian mentoring rely on changes to funding structures and attitudes toward research. There was insufficient evidence to confidently prescribe a best practice model. Sufficient frequency of qualitative reporting between publications concluded that mentoring is a valuable empowerment strategy in the areas of health and wellbeing, education and employment and as a remedial and preventative measure in reducing offending behaviours. An evidence-informed mentoring model would take into account the key findings of the review.

Keywords: Aboriginal and Torres Strait Islanders, Evidence, Indigenous Australians, Mentoring, Social determinants of health, Systematic review

\footnotetext{
* Correspondence: roxanne.bainbridge@jcu.edu.au

${ }^{1}$ The Cairns Institute, James Cook University, PO Box 6811, Cairns 4870,

Australia

Full list of author information is available at the end of the article
}

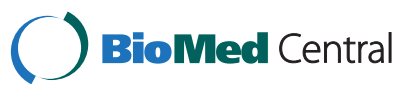

(c) 2014 Bainbridge et al.; licensee BioMed Central. This is an Open Access article distributed under the terms of the Creative Commons Attribution License (http://creativecommons.org/licenses/by/2.0), which permits unrestricted use, distribution, and reproduction in any medium, provided the original work is properly credited. The Creative Commons Public Domain Dedication waiver (http://creativecommons.org/publicdomain/zero/1.0/) applies to the data made available in this article, unless otherwise stated. 


\section{Background}

The pursuit of ways to positively influence the social determinants of health to improve health outcomes is a global mission. Viewed as a social determinant itself, empowerment $\mathrm{t}^{\mathrm{a}}$ and its associated strategies have become progressively evident in public health as a means of tackling the issue and equalising increasing health disparities [1]. Empowerment has application to broader populations and issues in public health, but evidence of its potential to improve a range of social determinants, health status and diverse health outcomes for socially-excluded populations is also increasing globally [1,2]. One change strategy, mentoring, is a firm predictor of resilience and empowerment [3] and can mitigate against those circumstances that contribute to a range of underlying determinants of health inequities. Adequately implemented, mentoring assists people to build personal resilience and life competences relative to needs through strengthening support networks [4-6]. It specifically involves "the commitment of time and specific efforts by a more experienced person to the development of a mutually beneficial, supportive and nurturing relationship with a less experienced person" [7]. The landscape of mentoring literature is vast. But despite the escalating literature base on mentoring in Western contexts over recent decades [8], growth in mentoring for diverse populations is a much newer area of study, and is not nearly as abundant.

Although there has been substantial international theorisng of mentoring processes, in the Aboriginal and Torres Strait Islander Australian context, these have been piecemeal. In Australia, there is a need to recognise the distinctiveness of social, economic and political systems and unique historical circumstances of Aboriginal and Torres Strait Islander (respectfully hereafter Indigenous) Australians. Dissenting epistemologies between Indigenous and other Australians infers the likelihood of key points of difference in mentoring standards. The need to recognise differences is also supported by the fact that strategies and programs found effective in working with Western populations have not enjoyed the same success when implemented with Indigenous Australian populations [9]. It holds therefore, that in contemplating mentoring work with Indigenous Australians, acknowledging Indigeneity is a particularly important consideration because at its heart are strong values-based connections that are shared in the ensuing relationships.

Identifying the best available evidence is critical to providing a baseline for future practice, policy and research. The stronger the evidence-base that exists in a particular field, the greater likelihood that the best responses can be understood and appropriate innovations of practice can be developed and transferred in a more time-oriented manner. Conversely, progress in a field of interest can be hampered by poor quality and inaccessibility of evidence.
Sanson-Fisher et al. [10] notes that the extent to which it is possible to use the best evidence to guide development in a field "will depend on the quantity and quality of available evidence", and, that "the number of measurement, descriptive and intervention research publications across time" can indicate whether research efforts have moved beyond describing the issue to providing data about how to facilitate change. Following Sanson-Fisher et al. [10], in this article, we systematically investigated the state of the evidence of mentoring literature for Indigenous Australians with the aim of: 1) ascertaining the quantity, nature and quality of relevant published documentation across time; and 2) providing some grounding for improving the evidence-base of mentoring for Indigenous Australians by identifying the nurturing grounds for the formation of mentoring relationships and the subsequent outcomes. The purpose of the latter was to develop a general structure of Indigenous Australian mentoring that describes different forms of mentoring relationships and explains what works best for successful outcomes. The review was initially prompted by an Australian government department, who, rather than starting from scratch to continually develop and implement new local solutions to Indigenous community issues, elected to work with university researchers to understand what was already working for Indigenous people in this area. The target population was urban-dwelling Indigenous youth and families; the aim of developing and implementing the mentoring program was to improve their access to education, employment, health and housing opportunities. The overall implications of the findings for the development of mentoring initiatives for Indigenous Australians are discussed. The review has therefore gone some way to linking the characteristics of mentoring as a strategy for addressing the social determinants of health through social inclusion, engagement and empowerment and provides a significant contribution to the public health literature.

\section{Methods}

The methodology of Sanson-Fisher et al. [10] informed the design of this systematic review. It also aligned with the approach of our five previous reviews [11-15]. We systematically appraised peer-reviewed and grey literature publications on mentoring for Indigenous Australians for the period January 1983 to October 2012; the start date coinciding with time mentoring literature entered academic writing and Kram's [16] seminal research on mentoring.

\section{Aim and objectives}

The overarching aim of the review was to: report on the state of evidence about mentoring initiatives for Indigenous Australian populations including children, 
young people and families. In the review, we critically appraised publications by:

- Taking account of the quantity of publications;

- Cataloguing publications according to nature/type;

- Mapping changes in publication outputs across the specified timeframes;

- Assessing the quality of publications; and

- Identifying the initiating environments; the facilitating environments; mentoring strategies; and mentoring outcomes.

\section{Search strategy}

As outlined in Figure 1, a five-step systematic review method was adopted. The five-steps are described in detail below.

\section{Step 1: Establishing the boundary definitions of mentoring}

It is difficult to gauge the effectiveness of a social innovation without defining its boundaries. For this reason, steps were taken to communicate the limits of mentoring for the purpose of this review. The boundary conditions follow the definition of mentoring articulated by Bozeman and Feeney [17] who define mentoring as: "a process for the informal transmission of knowledge, social capital and the psychosocial support perceived by the recipient as relevant...mentoring entails informal communication, usually face-to-face and during a sustained period of time, between a person who is perceived to have greater relevant knowledge, wisdom, or experience (the mentor) and a person who is perceived to have less (the protégé)". We also took account of limitations defined by Tolan et al. [18]. The boundaries for mentoring were:

1. There was an expectation of a trusting and mutually respectful relationship developing between the mentor and protégé or mentee over an extended timeframe irrespective of whether it was formally or informally developed;
2. In the relationship, mentors held more advanced knowledge and/or experience than the mentee and were committed to providing upward mobility and support to mentees according to mentees' needs;

3. The relationship involved the transmission of knowledge, social capital and psychosocial support according to the needs of mentees with the intention of fostering their growth and development; and

4. There was an absence of role inequality based in in the contexts of training, certification, parent-child or teacher-student relationships [18].

All publications involving Indigenous Australians children, young people, adults and families - in mentoring initiatives were included in the search. Any mentoring innovation for any mentor or mentee was studied, regardless of the setting. We included peer mentoring, group mentoring and individual mentoring, provided the activity reported a significant mentoring component that met the study description of mentoring.

\section{Step 2: A desktop canvassing of the literature}

A desktop analysis of literature pertaining to Indigenous Australian mentoring was conducted. We aimed to incorporate multidisciplinary literature sources in the data collection. Thirteen online databases Informit, Infotrac, Blackwell Publishing, Scopus, Web of Science, Proquest, Wiley, Taylor and Francis, JStor, the Australian Indigenous HealthInfoNet, Closing the Gap Clearinghouse, Google Scholar and Google were systematically searched by one author. Google and Google Scholar were searched to maximise search coverage of the grey literature. The first 100 returns of each, as per the Campbell Collaboration protocol for relevance and practicality (Personal Communication Campbell Collaboration, 2012) were included in the review. Reference lists of the final search documents were also scanned.

The search terms included mentor* plus Aborigin* or Torres or Indigenous. All identified publications were entered into Endnote. We searched the literature using

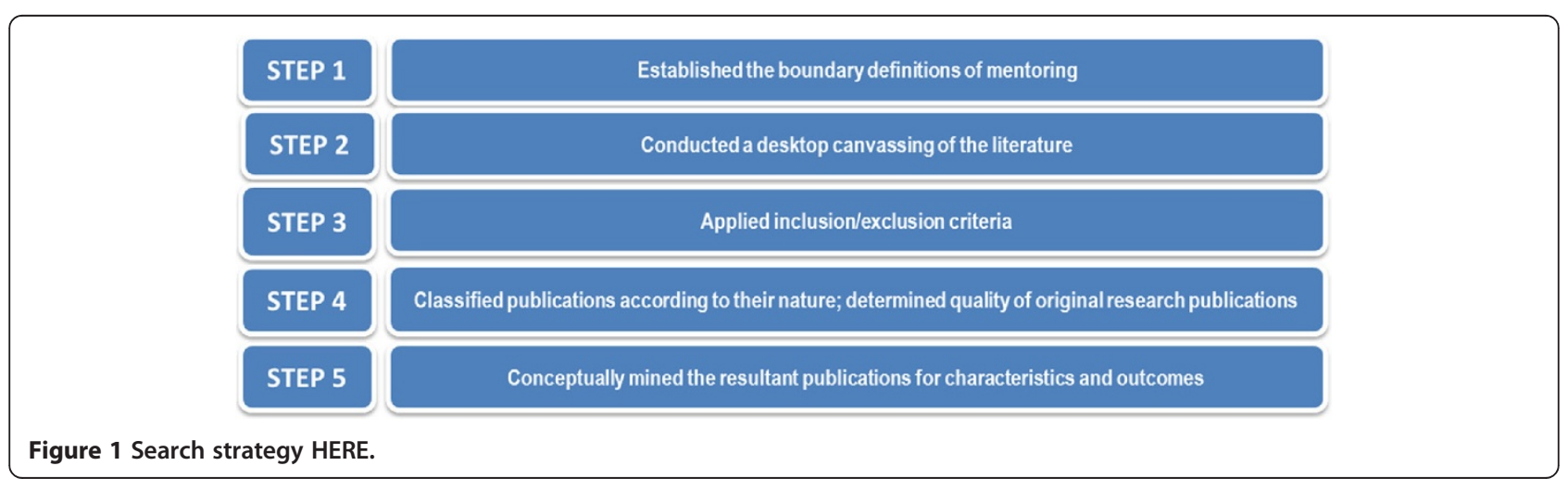


the term mentor* only; and did not search analogous terms. Terms potentially subsumed under the rubric of mentoring, such as coaching or role-modelling for instance, were not included because of fundamental differences in their methodological approaches to the implementation of such strategies and the nature of the experiences we sought to identify. It was important to understand how mentoring is at variance with other similar interpersonal relationships and the conditions under which it is implemented and described. Haggard et al. [19] highlight the importance of this assertion: "If agreement can be reached on the fundamental, distinctive attributes that set mentoring apart from other interpersonal relationships, then researchers can incorporate boundary conditions and the issue becomes what type of mentor[ing] is being studied". In coming to this conclusion, we examined the differences between mentoring and coaching and decided that mentoring differs from coaching in that coaching involves a more formal arrangement than mentoring with greater focus on tuition in specific experiences to achieve a defined goal in a controlled timeframe [20]. To confirm our decision, we also conducted a search for 'coaching' in Proquest for no returns. However, we acknowledge the potential overlapping nature of these terms and those identified in the concept of mentoring. For instance, coaching for particular a skillset is often incorporated under mentoring strategies.

\section{Step 3: Applying inclusion/exclusion criteria}

Inclusion criteria developed at the outset of the search were applied to the retrieved documents. Publications were included if:

- The key search terms were located in the title or abstract;

- Documents were available in English;

- Documents were published between January 1983 and October 2012;

- Documents explicitly referred to experiences as 'mentoring';

- Documents identified Aboriginal and/or Torres Strait Islander Australians and mentoring as their key focus of concern (according to the above components of mentoring); and

- Documents compared Aboriginal and/or Torres Strait Islander Australians to other groups.

We excluded publications where the mentoring process or the effects of the mentoring innovation could not be separated from other innovations.

\section{Step 4: Classification of publications}

The inclusion/exclusion process procured 15 final review publications. We then implemented a three-phased approach to classify their nature. In Phase 1, publications were classified according to the type of publication; in Phase 2 original research publications were classified to the domains of measurement, descriptive or intervention research; in Phase 3, inter-rater reliability was established; and in Phase 4, the quality of study publications was assessed.

Phase 1: Publications were grouped according to publication type as identified by Sanson-Fisher et al. [10]: original research, reviews, program descriptions, discussion papers/commentaries or case reports.

Phase 2: Original research publications were then classified under three categories: measurement, descriptive and intervention research:

- Measurement research: Publications that developed or tested a measure of mentoring for use in Indigenous Australian populations, or a measure concerned with Indigenous Australian issues;

- Descriptive research: Publications where the primary aim was to explore issues, processes/models or attributes related to mentoring;

- Intervention research: Publications in which the aim was to test the effectiveness of an intervention implemented with Indigenous Australians, such as wellbeing/empowerment, education and employment in mentoring programs. This category included research where the aim was to examine the impact of interventions designed to alter knowledge, attitudes or behaviours or to improve program delivery.

Phase 3: Thirty-seven (37/771 or $4.8 \%)$ publications were identified for full-text review. A subset of 19 publications (19/37 or $51.3 \%$ was blindly assessed by another author (a stakeholder in a mentoring program) to verify inclusion and classification of publications selected by the initial researcher. There was $79 \%$ agreement. Full consensus was then reached in negotiations between those two authors on the final decision of fifteen included publications.

Phase 4: Quality was determined using two indicators: 1) methodological quality; and 2) peer-review. Research evidence is commonly assessed by the methodological quality by which it is generated. The quality of original research publications was assessed and rated as strong, moderate or weak using the Critical Appraisal Skills Programme (CASP)'s [21] appraisal checklists for qualitative studies. Peer-review increases the probability of quality [10] and as such was used as a benchmark.

\section{Step 5: Conceptually mining the data}

The characteristics and outcomes of mentoring were identified by conceptually mining the 15 resultant publications according to a predefined framework. This was 
achieved by hand-searching each publication for the framework elements. These included: author; publication year; publication type; type of program; program location; target population; numbers of participants; program aims; publication classifications; quality of the design for original research publications; initiating environments; facilitating environments; operational strategies; and mentoring outcomes. Initiating environments were defined as the boundaries within which mentoring initiatives were developed; facilitating environments, the operational elements of mentoring practice; operational strategies, the mechanisms that underlie mentoring relationships; and outcomes, those which were subsequential to Indigenous Australian experiences of mentoring.

\section{Results}

Figure 2 is a flow chart [22] of how the total number of publications identified was reduced to the final fifteen publications focussed on Indigenous Australian mentoring. In Table 1, the classifications and quality of the selected original research publications are displayed in Table 2.
The quantity, nature and quality of identified publications Number of publications

A total of 771 publications were identified and included a screening of the first 100 each in Google $(n=132,000)$ and Google Scholar $(n=16,800)$. Three additional records were identified through searching references and one through a social networking site. Duplicates numbering 148 were then removed leaving 623 records. After screening titles and abstracts, 586 records were removed for not meeting the inclusion criteria; this left 37 fulltext records to be more closely assessed. Fifteen of the 37 records were eligible. There were no publications from 1983 to 1998 but publications increased thereafter. The fifteen publications spanned a timeframe from 1999 to 2012; increasing from 1 per annum from 1999 to 2008 , excepting $2005(\mathrm{n}=2)$, to two in 2009 , three for 2010 and four in 2011; and then to mid-2012 (at the time of the search) only 1 paper had been published (See Figure 3).

\section{Classification of publications}

Publications included: case reports $(n=6)$, journal articles $(n=4)$, web pages $(n=3)$ and conference papers $(\mathrm{n}=2)$. Fourteen/15 (or 92.8\%) documents were classified

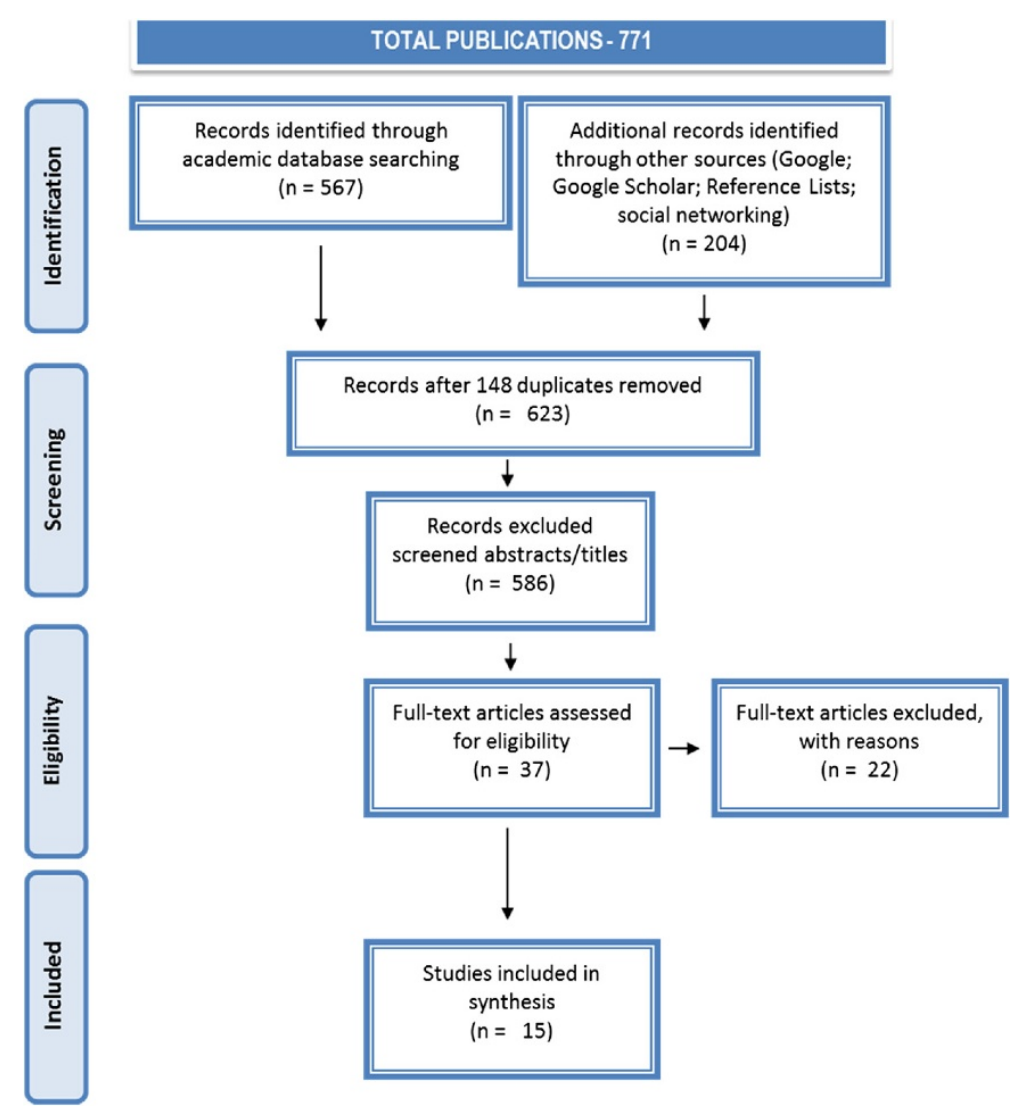

Figure 2 Flow diagram indigenous Australian mentoring HERE. 
Table 1 Nature of classifications

\begin{tabular}{|c|c|c|c|c|c|c|c|}
\hline Year & All & Measurement & Descriptive & Intervention & Review & Program Description & Case Report \\
\hline 1999 & 1 & - & - & 1 & - & - & - \\
\hline 2000 & - & - & - & - & - & - & - \\
\hline 2001 & - & - & - & - & - & - & - \\
\hline 2002 & - & - & - & - & - & - & - \\
\hline 2003 & - & - & - & - & - & - & - \\
\hline 2004 & 1 & - & 1 & - & - & - & - \\
\hline 2005 & 2 & - & 1 & - & - & 1 & - \\
\hline 2006 & - & - & - & - & - & - & - \\
\hline 2007 & - & - & - & - & - & - & - \\
\hline 2008 & 1 & - & 1 & - & - & - & - \\
\hline 2009 & 2 & - & 1 & - & - & - & 1 \\
\hline 2010 & 3 & - & - & - & 1 & 1 & 1 \\
\hline 2011 & 4 & - & - & - & - & 3 & 1 \\
\hline 2012 & 1 & - & - & - & - & 1 & - \\
\hline Total & 15 & - & 4 & 1 & 1 & 6 & 3 \\
\hline
\end{tabular}

as descriptive. These were counted as program descriptions $(n=6)$ [23-28], descriptive original research papers $(n=5)$ [29-33], descriptive case reports $(n=3)$ [34-36]; and a review of mentoring programs $(n=1)$ [37]. Only four documents were peer-reviewed publications $[23,26,28,31]$. Original research publications were then classified under three categories: measurement $(n=0)$, descriptive $(\mathrm{n}=4)[29,31-33]$.

\section{Quality of publications}

Because all studies were qualitative, the overall methodological quality of those publications classified as original research $(n=5)$ [29-33] were appraised using the CASP tool for qualitative study appraisal [21]. The overall rating of the methodological quality of original research studies was generally strong: four/five studies used strong research designs [29-31,33]; with one rated as using a weak design [33]. Three/4 of the stronger designs were descriptive program evaluations. Methodological deficiencies of the study using the weak design included failure to adequately articulate research processes in terms of researcher relationships, ethical issues, rigorous analysis, supporting data and research value. Only one paper [29] met both quality criteria of methodological quality.

\section{The characteristics of mentoring}

Table 3 displays the key characteristics of the fifteen resultant publications; while Figure 4 provides a succinct summary of the characteristics in terms of initiating environments, facilitating environments, strategies and outcomes of mentoring.

\section{Initiating environments}

The fifteen eligible publications focussed on Indigenous Australian mentoring implemented in various places across Australia. Mentoring approaches encompassed a range of

Table 2 The quality of indigenous Australian mentoring publications 1983 - 2012: original research publications

\begin{tabular}{|c|c|c|c|}
\hline Author/Publication year & Publication type & Publication classification & Quality of study design \\
\hline \multirow[t]{2}{*}{ Burgess \& Dyer, 2009} & Journal Article & Original Research & Methodological: Strong Peer-reviewed \\
\hline & & Descriptive & \\
\hline \multirow[t]{2}{*}{ Nasir, 2008} & Conference Paper & Original research & Methodological: Weak \\
\hline & & Descriptive & \\
\hline \multirow[t]{2}{*}{ MacCallum, Beltman \& Palmer, 2005} & Conference Paper & Original Research & Methodological: Strong \\
\hline & & Descriptive & \\
\hline \multirow[t]{2}{*}{ Stacey, 2004} & Report & Original Research & Methodological: Strong \\
\hline & & Descriptive & \\
\hline \multirow[t]{2}{*}{ Department of Local Government, 1999} & Report & Original Research & Methodological: Strong \\
\hline & & Intervention Research & \\
\hline
\end{tabular}




\section{Publication Outputs}

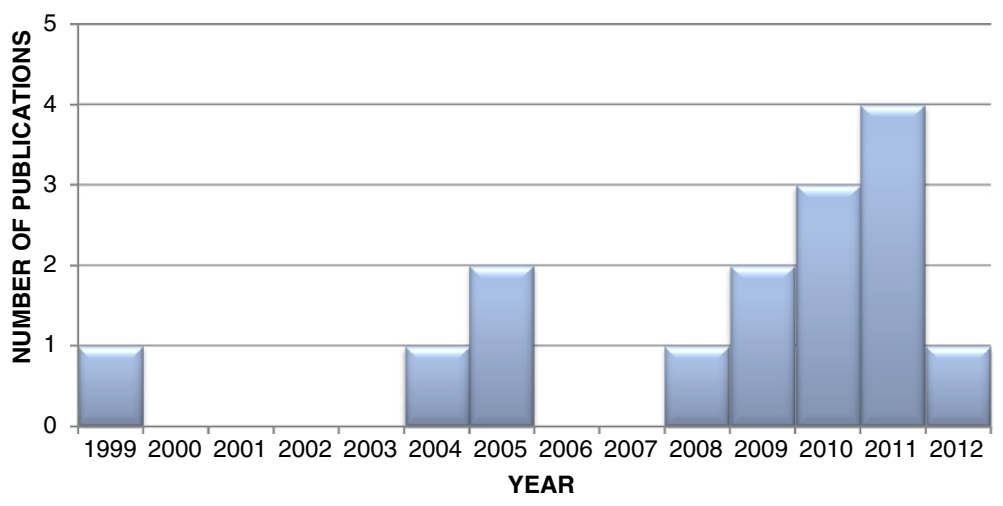

Figure 3 Publication outputs over time HERE.

program designs that addressed specific issues and subsets of the Indigenous Australian population. They were spread across several different contexts: workplace $[n=4]$, at-risk youth $[n=3]$, secondary education $[n=6]$, health promotion $[n=1]$ and political participation $[n=1]$. Nine $(60 \%)$ publications were concentrated on youth mentoring.

Most publications were centred on mentoring programs that aimed to improve education and employment outcomes for Indigenous Australians (11/15 or $80 \%)$. This focus was in sharp contrast to publications (3/15 or $20 \%)$ described as program innovations to address specific risk areas or problem behaviours, for example, changing offending behaviours for 'at-risk' youth [33]; young offenders [23]; and post-release young people [28]. Programs with educational and employment goals accounted for 11/15 (73.3\%) publications: there were six (40\%) secondary school-based mentoring programs documented and five (33\%) workplace initiatives. School-based programs expanded rapidly over the past seven years, from one in 2005, to five being developed from 2009 to 2012. Sitebased programs in the workplace were initiated in 1999; the other four more recent publications were spread from 2008 to 2010. Thus academic and employment-related aims were driving most of the programs. Only three/15 publications $[28,31,33]$ set attitude change as part of their explicit aims. The first sought change in the expectations of success for Indigenous Australian students for students, families and teachers; and the second, to promote selfdiscovery and self-determination; and the last sought to encourage self-respect and independence.

\section{Facilitating environments}

The facilitating environments, in which mentoring relationships developed, incorporated the operational elements of mentoring practice. Varied participant numbers were found across publications reporting figures in mentoring programs. They ranged from smaller programs with
10 mentees [32], 13 mentees [29] and 14 mentees [27] to 566 mentees [36]. The largest numbers were evidenced in three school-based programs: PASS Australia [26] had 525 mentees; AIME [34-36] had 483 mentees, 366 and 566 respectively; and there were 483 mentees in the National Indigenous Mentoring Pilots Program [31]. In all cases these participant numbers were distributed across a number of sites, thus reducing the number of participants at each site significantly. For example, 483 mentees in the National Indigenous Mentoring Pilots Program was spread across 53 sites; therefore averaging 9 mentees per site.

All publications reported planned and systematic organisation of mentoring practices; nevertheless, in 9/15 or $60 \%$ publications voluntarily participation of mentees in programs were reported; the nature of participation for mentees was not mentioned in all other cases. The skills, qualities and competencies required by mentors to fulfil their commitments to Indigenous Australian mentees were taken into account. Eight/15 (53.3\%) publications described having mentor training [23,26-29,31,33,37]; seven of these included cultural awareness training as one aspect of their training. The Tribal Warriors Program [28] developed locally-tailored mentoring accreditation as part of their program. Another, Nasir [32] critiqued the incidence of untrained mentors. In all cases, the mentor was considerably more knowledgeable than the mentee.

The matching of mentors to mentees either in terms of mutual connections [30], gender and ethnicity [29]; socio-economic and cultural background [25] and/or areas of interest/expertise [23,25] according to mentee needs $[25,27]$ was evidenced in five cases. In one instance, mentees had a choice of mentors [23]. In other cases, mentors were either not matched to mentees, or this was not reported. While many publications [23,25,27,29,31,34-36], $8 / 15$ or $53.3 \%$, stated that mentors volunteered, $4 / 15$ or $26.6 \%$ publications $[26,28,32,33]$ reported employing paid mentors in their programs; and three cases [24,30,37] did 
Table 3 The key characteristics of Indigenous Australian mentoring publications 1983 - 2012

\begin{tabular}{|c|c|c|c|c|c|c|c|c|}
\hline $\begin{array}{l}\text { Author/publication } \\
\text { Year/publication type }\end{array}$ & Type of program & $\begin{array}{l}\text { Program } \\
\text { location }\end{array}$ & Target population & $\begin{array}{l}\text { No. of } \\
\text { participants }\end{array}$ & Program aim & Mentoring strategies & $\begin{array}{l}\text { Publication } \\
\text { classification/ } \\
\text { Quality of } \\
\text { study design }\end{array}$ & Outcomes \\
\hline \multirow[t]{11}{*}{$\begin{array}{l}\text { Dawes \& Dawes, } \\
2005 \text { Journal Article }\end{array}$} & \multirow{11}{*}{$\begin{array}{l}\text { Mentoring } \\
\text { program for } \\
\text { young Aboriginal } \\
\text { and Torres Strait } \\
\text { Islander offenders }\end{array}$} & $\begin{array}{l}\text { Detention } \\
\text { Centre }\end{array}$ & \multirow{11}{*}{$\begin{array}{l}\text { Young Aboriginal and } \\
\text { Torres Strait Islander } \\
\text { men }\end{array}$} & \multirow[t]{11}{*}{48} & \multirow{2}{*}{$\begin{array}{l}\text { Successful transition of } \\
\text { students back into their } \\
\text { family and wider } \\
\text { communities }\end{array}$} & $\begin{array}{l}\text { Mentors trained, including } \\
\text { cultural awareness }\end{array}$ & \multirow[t]{11}{*}{$\begin{array}{l}\text { Program } \\
\text { description }\end{array}$} & \multirow[t]{11}{*}{ Positive relationships } \\
\hline & & \multirow{10}{*}{$\begin{array}{l}\text { Far North } \\
\text { Queensland }\end{array}$} & & & & Volunteer mentors & & \\
\hline & & & & & \multirow{9}{*}{$\begin{array}{l}\text { Reduce reoffending } \\
\text { behaviour and establish } \\
\text { links to further } \\
\text { education and } \\
\text { employment pathways }\end{array}$} & Mentee choice of mentors & & \\
\hline & & & & & & Matched on interests & & \\
\hline & & & & & & One-on-one weekly & & \\
\hline & & & & & & Role-modelling & & \\
\hline & & & & & & Listening & & \\
\hline & & & & & & $\begin{array}{l}\text { Relationship building } \\
\text { activities e.g. chess, fishing }\end{array}$ & & \\
\hline & & & & & & $\begin{array}{l}\text { Literacy, numeracy; } \\
\text { vocationally-oriented } \\
\text { curriculum (building and } \\
\text { construction, art, } \\
\text { horticulture, hospitality) }\end{array}$ & & \\
\hline & & & & & & $\begin{array}{l}\text { Links to, and support of } \\
\text { Education Queensland }\end{array}$ & & \\
\hline & & & & & & $\begin{array}{l}\text { Funding Education } \\
\text { Queensland and Cleveland } \\
\text { Education \& Training } \\
\text { Centre }\end{array}$ & & \\
\hline \multirow[t]{9}{*}{$\begin{array}{l}\text { Burgess \& Dyer, } 2009 \\
\text { Journal Article }\end{array}$} & \multirow{9}{*}{$\begin{array}{l}\text { Workplace } \\
\text { Mentoring } \\
\text { Program }\end{array}$} & \multirow{9}{*}{$\begin{array}{l}\text { University of } \\
\text { Newcastle } \\
\text { NSW }\end{array}$} & \multirow[t]{9}{*}{$\begin{array}{l}\text { Indigenous Australian } \\
\text { university trainees }\end{array}$} & \multirow[t]{9}{*}{13} & \multirow{9}{*}{$\begin{array}{l}\text { Assist Indigenous job } \\
\text { seekers enter the } \\
\text { mainstream workforce }\end{array}$} & \multirow{3}{*}{$\begin{array}{l}12 \text { month Indigenous paid } \\
\text { traineeship: combination of } \\
\text { study, hands-on-learning } \\
\text { and formal and peer } \\
\text { mentoring }\end{array}$} & $\begin{array}{l}\text { Original } \\
\text { Research }\end{array}$ & University Certificates. \\
\hline & & & & & & & Descriptive & \multirow{8}{*}{$\begin{array}{l}12 / 20 \text { completions: } 8 / 20 \\
\text { completions with } \\
\text { university employment; } \\
4 / 20 \text { completions with } \\
\text { external university } \\
\text { employment }\end{array}$} \\
\hline & & & & & & & \multirow[t]{7}{*}{ Strong } & \\
\hline & & & & & & $\begin{array}{l}\text { Trained mentors (cultural } \\
\text { awareness) }\end{array}$ & & \\
\hline & & & & & & One-on-one mentoring & & \\
\hline & & & & & & $\begin{array}{l}\text { Mentors matched to } \\
\text { gender and ethnicity }\end{array}$ & & \\
\hline & & & & & & Voluntary participation & & \\
\hline & & & & & & $\begin{array}{l}\text { Flexible to the needs of } \\
\text { mentees }\end{array}$ & & \\
\hline & & & & & & $\begin{array}{l}\text { Partnerships with local } \\
\text { Indigenous communities } \\
\text { and community } \\
\text { organisations }\end{array}$ & & \\
\hline
\end{tabular}




\begin{tabular}{|c|c|c|c|c|c|c|c|c|}
\hline & & & & & & Volunteer external mentors & & \\
\hline & & & & & & $\begin{array}{l}\text { Psychological, role- } \\
\text { modelling, counselling, } \\
\text { acceptance/confirmation, } \\
\text { friendship, career } \\
\text { development }\end{array}$ & & \\
\hline & & & & & & $\begin{array}{l}\text { Funding the University of } \\
\text { Newcastle }\end{array}$ & & \\
\hline \multirow{13}{*}{$\begin{array}{l}\text { Paase \& Adams, } 2011 \\
\text { Journal Article }\end{array}$} & \multirow{13}{*}{$\begin{array}{l}\text { Indigenous peer } \\
\text { mentoring } \\
\text { program }\end{array}$} & \multirow{2}{*}{$\begin{array}{l}\text { Chronic } \\
\text { disease } \\
\text { prevention }\end{array}$} & \multirow{13}{*}{$\begin{array}{l}\text { Indigenous people } \\
\text { living in the inner } \\
\text { suburbs of west } \\
\text { Melbourne }\end{array}$} & \multirow[t]{13}{*}{ Not reported } & \multirow{13}{*}{$\begin{array}{l}\text { Developing a mentoring } \\
\text { model to improve the } \\
\text { health of Indigenous } \\
\text { people }\end{array}$} & \multirow{2}{*}{$\begin{array}{l}\text { Consultation with Local } \\
\text { Indigenous people }\end{array}$} & \multirow{3}{*}{$\begin{array}{l}\text { Program } \\
\text { Description }\end{array}$} & Smoking cessation \\
\hline & & & & & & & & Skill acquisition \\
\hline & & \multirow[t]{11}{*}{ Melbourne } & & & & $\begin{array}{l}\text { Voluntary participation } \\
\text { (mentees) }\end{array}$ & & \multirow[t]{2}{*}{$\begin{array}{l}\text { Increased expression of } \\
\text { identity }\end{array}$} \\
\hline & & & & & & Built on existing strengths & & \\
\hline & & & & & & $\begin{array}{l}\text { Tailored to local needs and } \\
\text { culture }\end{array}$ & & \multirow[t]{9}{*}{$\begin{array}{l}\text { Reduction in cultural } \\
\text { isolation }\end{array}$} \\
\hline & & & & & & $\begin{array}{l}\text { Local knowledge linked } \\
\text { with existing services and } \\
\text { programs }\end{array}$ & & \\
\hline & & & & & & $\begin{array}{l}\text { Partnerships with } \\
\text { organisations }\end{array}$ & & \\
\hline & & & & & & Group mentoring & & \\
\hline & & & & & & $\begin{array}{l}\text { Indigenous program team, } \\
\text { peer mentors and peers } \\
\text { locally employed }\end{array}$ & & \\
\hline & & & & & & $\begin{array}{l}\text { Formal \& informal } \\
\text { mentoring }\end{array}$ & & \\
\hline & & & & & & Volunteer mentors & & \\
\hline & & & & & & $\begin{array}{l}\text { Trained peer mentors (as } \\
\text { mentors and skill } \\
\text { development); ongoing } \\
\text { support }\end{array}$ & & \\
\hline & & & & & & $\begin{array}{l}\text { Matched cultural/socio- } \\
\text { economic background and/ } \\
\text { or interests }\end{array}$ & & \\
\hline \multirow[t]{4}{*}{$\begin{array}{l}\text { Tribal Warriors, } 2011 \\
\text { Journal Article }\end{array}$} & \multirow{4}{*}{$\begin{array}{l}\text { Post-release } \\
\text { mentoring } \\
\text { program for } \\
\text { young Aboriginal } \\
\text { offenders }\end{array}$} & \multirow{4}{*}{$\begin{array}{l}\text { Redfern } \\
\text { Community } \\
\text { Redfern, } \\
\text { NSW }\end{array}$} & \multirow{4}{*}{$\begin{array}{l}\text { Aboriginal young } \\
\text { people ( } 7-25 \text { years) } \\
\text { recently released from } \\
\text { correctional centres }\end{array}$} & \multirow[t]{4}{*}{$\begin{array}{l}50 \\
\text { participants }\end{array}$} & \multirow{4}{*}{$\begin{array}{l}\text { Encourage education, } \\
\text { self-respect and inde- } \\
\text { pendence (post-release) } \\
\text { for young Aboriginal } \\
\text { offenders }\end{array}$} & $\begin{array}{l}\text { Mentors walk with mentees } \\
\text { through many aspects of }\end{array}$ & \multirow[t]{4}{*}{$\begin{array}{l}\text { Program } \\
\text { Description }\end{array}$} & \multirow{4}{*}{$\begin{array}{l}\text { Developed a mentoring } \\
\text { certificate course. } \\
\text { Decreased re-offending } \\
\text { including decrease of } \\
80 \% \text { in men charged } \\
\text { with robbery }\end{array}$} \\
\hline & & & & & & One-on-one mentoring & & \\
\hline & & & & & & Elder leadership & & \\
\hline & & & & & & $\begin{array}{l}\text { Elder and police } \\
\text { collaboration }\end{array}$ & & \\
\hline
\end{tabular}




\begin{tabular}{|c|c|c|c|c|c|c|c|c|}
\hline & & & & & & $\begin{array}{l}\text { Police-mentee and police- } \\
\text { community liaison }\end{array}$ & & $\begin{array}{l}\text { Improved relationships } \\
\text { between police and the }\end{array}$ \\
\hline & & & & & & Paid mentors & & \\
\hline & & & & & & $\begin{array}{l}\text { Mentors trained, including } \\
\text { cultural awareness }\end{array}$ & & \\
\hline & & & & & & $\begin{array}{l}\text { Training, employment and } \\
\text { education opportunities e. } \\
\text { g. Certified maritime } \\
\text { training }\end{array}$ & & \\
\hline & & & & & & $\begin{array}{l}\text { Health and wellbeing } \\
\text { training including family } \\
\text { violence, substance abuse, } \\
\text { fitness }\end{array}$ & & \\
\hline & & & & & & Cultural participation & & \\
\hline & & & & & & $\begin{array}{l}\text { Funded by Department of } \\
\text { Community Services; } \\
\text { Department of Families, } \\
\text { Housing, Community } \\
\text { Services and Indigenous } \\
\text { Affairs; Centrelink; } \\
\text { Department of Education, } \\
\text { Employment and } \\
\text { Workplace Relations }\end{array}$ & & \\
\hline \multirow[t]{7}{*}{$\begin{array}{l}\text { Nasir, } 2008 \\
\text { Conference Paper }\end{array}$} & \multirow{7}{*}{$\begin{array}{l}\text { Mentoring } \\
\text { program for } \\
\text { Indigenous } \\
\text { apprentices }\end{array}$} & \multirow{7}{*}{$\begin{array}{l}\text { Group } \\
\text { training } \\
\text { organisation } \\
\text { - public } \\
\text { sector Not } \\
\text { reported }\end{array}$} & \multirow[t]{7}{*}{ Indigenous apprentices } & \multirow{7}{*}{$\begin{array}{l}10 \text { Indigenous } \\
\text { apprentices, } 4 \\
\text { field officers, } 2 \\
\text { group training } \\
\text { organisation } \\
\text { managers, } 3 \\
\text { host employer } \\
\text { supervisors } \\
\text { and } 1 \text { trainer }\end{array}$} & \multirow{7}{*}{$\begin{array}{l}\text { To improve the } \\
\text { recruitment, retention } \\
\text { and completion of } \\
\text { apprenticeships by } \\
\text { Indigenous Australians }\end{array}$} & $\begin{array}{l}\text { Mentors recruited into the } \\
\text { organisation }\end{array}$ & $\begin{array}{l}\text { Original } \\
\text { research }\end{array}$ & \multirow[t]{7}{*}{$\begin{array}{l}\text { Critiqued for being } \\
\text { ineffective }\end{array}$} \\
\hline & & & & & & & Descriptive & \\
\hline & & & & & & $\begin{array}{l}\text { Untrained, unsupported } \\
\text { mentors }\end{array}$ & Weak & \\
\hline & & & & & & Appointed mentors & & \\
\hline & & & & & & $\begin{array}{l}\text { Mentors have dual roles in } \\
\text { the organisation }\end{array}$ & & \\
\hline & & & & & & One-on-one mentoring & & \\
\hline & & & & & & $\begin{array}{l}\text { Funding local training } \\
\text { organisation }\end{array}$ & & \\
\hline \multirow{6}{*}{$\begin{array}{l}\text { MacCallum, Beltman } \\
\text { \& Palmer, 2005 } \\
\text { Conference Paper }\end{array}$} & \multirow{6}{*}{$\begin{array}{l}\text { National } \\
\text { Indigenous } \\
\text { Mentoring Pilots } \\
\text { Project }\end{array}$} & \multirow{6}{*}{$\begin{array}{l}\text { Secondary } \\
\text { Schools; } \\
\text { detention } \\
\text { centres; } \\
\text { residential } \\
\text { schools } 53 \\
\text { sites across } \\
\text { Australia }\end{array}$} & \multirow{6}{*}{$\begin{array}{l}\text { Indigenous high school } \\
\text { students }\end{array}$} & \multirow{6}{*}{$\begin{array}{l}53 \text { sites } 483 \\
\text { mentees } 332 \\
\text { mentors }\end{array}$} & \multirow{4}{*}{$\begin{array}{l}\text { To trial mentoring } \\
\text { approaches to improve } \\
\text { literacy, numeracy, } \\
\text { attendance and } \\
\text { participation of high } \\
\text { school students. }\end{array}$} & One-on-one mentoring & \multirow{6}{*}{$\begin{array}{l}\text { Original } \\
\text { Research } \\
\text { Descriptive } \\
\text { Strong }\end{array}$} & \multirow{4}{*}{$\begin{array}{l}\text { Mentees: Increased } \\
\text { self-confidence and } \\
\text { self-esteem; school } \\
\text { attendance; retention; } \\
\text { and participation in } \\
\text { classroom tasks }\end{array}$} \\
\hline & & & & & & Trained volunteer mentors & & \\
\hline & & & & & & 1 hour per week & & \\
\hline & & & & & & \multirow{2}{*}{$\begin{array}{l}\text { Exposure to employment } \\
\text { pathways, community work } \\
\text { and further education }\end{array}$} & & \\
\hline & & & & & \multirow{2}{*}{$\begin{array}{l}\text { To raise students' } \\
\text { expectations of success } \\
\text { and the expectations of }\end{array}$} & & & \multirow{2}{*}{$\begin{array}{l}\text { Enhanced valuing of } \\
\text { school and connections }\end{array}$} \\
\hline & & & & & & Awards Celebrations & & \\
\hline
\end{tabular}


Table 3 The key characteristics of Indigenous Australian mentoring publications 1983 - 2012 (Continued)

\begin{tabular}{|c|c|c|c|c|c|c|c|c|}
\hline & & & & & \multirow{15}{*}{$\begin{array}{l}\text { their parents and } \\
\text { teachers }\end{array}$} & \multirow{2}{*}{\multicolumn{2}{|c|}{$\begin{array}{l}\text { Life skills } \\
\text { Role-modelling }\end{array}$}} & \multirow{2}{*}{$\begin{array}{l}\text { between school and } \\
\text { work }\end{array}$} \\
\hline & & & & & & & & \\
\hline & & & & & & \multicolumn{2}{|l|}{ Identity building } & \multirow{2}{*}{$\begin{array}{l}\text { Increased ability to } \\
\text { solve personal and } \\
\text { social problems }\end{array}$} \\
\hline & & & & & & \multicolumn{2}{|l|}{ Motivational speakers } & \\
\hline & & & & & & \multicolumn{2}{|l|}{$\begin{array}{l}\text { Social activities- fishing, } \\
\text { gardening, dance, art, sport, } \\
\text { camps }\end{array}$} & $\begin{array}{l}\text { Development of } \\
\text { leadership and life skills }\end{array}$ \\
\hline & & & & & & \multicolumn{2}{|l|}{ Reconciliatory approach } & \multirow{2}{*}{$\begin{array}{l}\text { Improved relationships } \\
\text { with, and between } \\
\text { peers, teachers and } \\
\text { family members }\end{array}$} \\
\hline & & & & & & \multicolumn{2}{|l|}{$\begin{array}{l}\text { Vocational educational } \\
\text { experiences }\end{array}$} & \\
\hline & & & & & & \multicolumn{2}{|l|}{ Recognition of Indigeneity } & \multirow{2}{*}{$\begin{array}{l}\text { Improved literacy and } \\
\text { numeracy }\end{array}$} \\
\hline & & & & & & \multicolumn{2}{|l|}{ Respectful relationships } & \\
\hline & & & & & & \multicolumn{2}{|l|}{ Mentor humour } & \multirow{5}{*}{$\begin{array}{l}\text { Mentors: improved } \\
\text { knowledge of } \\
\text { Indigenous culture and } \\
\text { youth issues; } \\
\text { development of strong } \\
\text { relationships with } \\
\text { students; enhanced } \\
\text { personal development } \\
\text { and self-esteem. }\end{array}$} \\
\hline & & & & & & \multicolumn{2}{|l|}{$\begin{array}{l}\text { Involvement of families and } \\
\text { communities }\end{array}$} & \\
\hline & & & & & & \multicolumn{2}{|l|}{ Genealogy program } & \\
\hline & & & & & & \multicolumn{2}{|l|}{$\begin{array}{l}\text { Networking for } \\
\text { employment }\end{array}$} & \\
\hline & & & & & & \multirow{2}{*}{\multicolumn{2}{|c|}{$\begin{array}{l}\text { Funding Department of } \\
\text { Education, Science \& } \\
\text { Training }\end{array}$}} & \\
\hline & & & & & & & & $\begin{array}{l}\text { School and Community: } \\
\text { enhanced links } \\
\text { between school and } \\
\text { community; increased } \\
\text { involved of families in } \\
\text { school; awareness of, } \\
\text { and access to local } \\
\text { Indigenous role-models; } \\
\text { development of inter- } \\
\text { school relationships; } \\
\text { positive contact } \\
\text { between Indigenous } \\
\text { and non-Indigenous } \\
\text { families. }\end{array}$ \\
\hline \multirow[t]{5}{*}{ Stacey, 2004 Report } & \multirow{5}{*}{$\begin{array}{l}\text { Panyappi } \\
\text { Indigenous youth } \\
\text { mentoring service }\end{array}$} & \multirow{5}{*}{$\begin{array}{l}\text { Indigenous } \\
\text { Youth } \\
\text { Mentoring } \\
\text { Program } \\
\text { South } \\
\text { Australia }\end{array}$} & \multirow{5}{*}{$\begin{array}{l}\text { Indigenous youth } \\
\text { 'at-risk' at of being a } \\
\text { victim of crime or } \\
\text { engaging in offending } \\
\text { behaviour. }\end{array}$} & \multirow{5}{*}{$\begin{array}{l}30 \text { Urban } \\
\text { (inner city) } \\
\text { Indigenous } \\
\text { youth 10-17 } \\
\text { years }\end{array}$} & \multirow{2}{*}{$\begin{array}{l}\text { To intervene in } \\
\text { pathways of offending } \\
\text { behaviour }\end{array}$} & $\begin{array}{l}\text { Works from a development } \\
\text { perspective }\end{array}$ & $\begin{array}{l}\text { Original } \\
\text { Research }\end{array}$ & \multirow[t]{2}{*}{$\begin{array}{l}\text { Marked change in } \\
\text { offending behaviour }\end{array}$} \\
\hline & & & & & & \multirow{3}{*}{$\begin{array}{l}\text { Family-inclusive approach } \\
\text { Accredited trained paid } \\
\text { mentors including cultural } \\
\text { awareness }\end{array}$} & Descriptive & \\
\hline & & & & & To decrease each young & & Strong & \multirow{3}{*}{$\begin{array}{l}\text { Attitude shift } \\
\text { Decreased frequency of } \\
\text { offending }\end{array}$} \\
\hline & & & & & $\begin{array}{l}\text { with the juvenile justice } \\
\text { system and/or agencies }\end{array}$ & & & \\
\hline & & & & & $\begin{array}{l}\text { associated with this } \\
\text { system. }\end{array}$ & \multicolumn{2}{|l|}{$\begin{array}{l}\text { Mentor support and } \\
\text { supervision }\end{array}$} & \\
\hline
\end{tabular}


Table 3 The key characteristics of Indigenous Australian mentoring publications 1983 - 2012 (Continued)

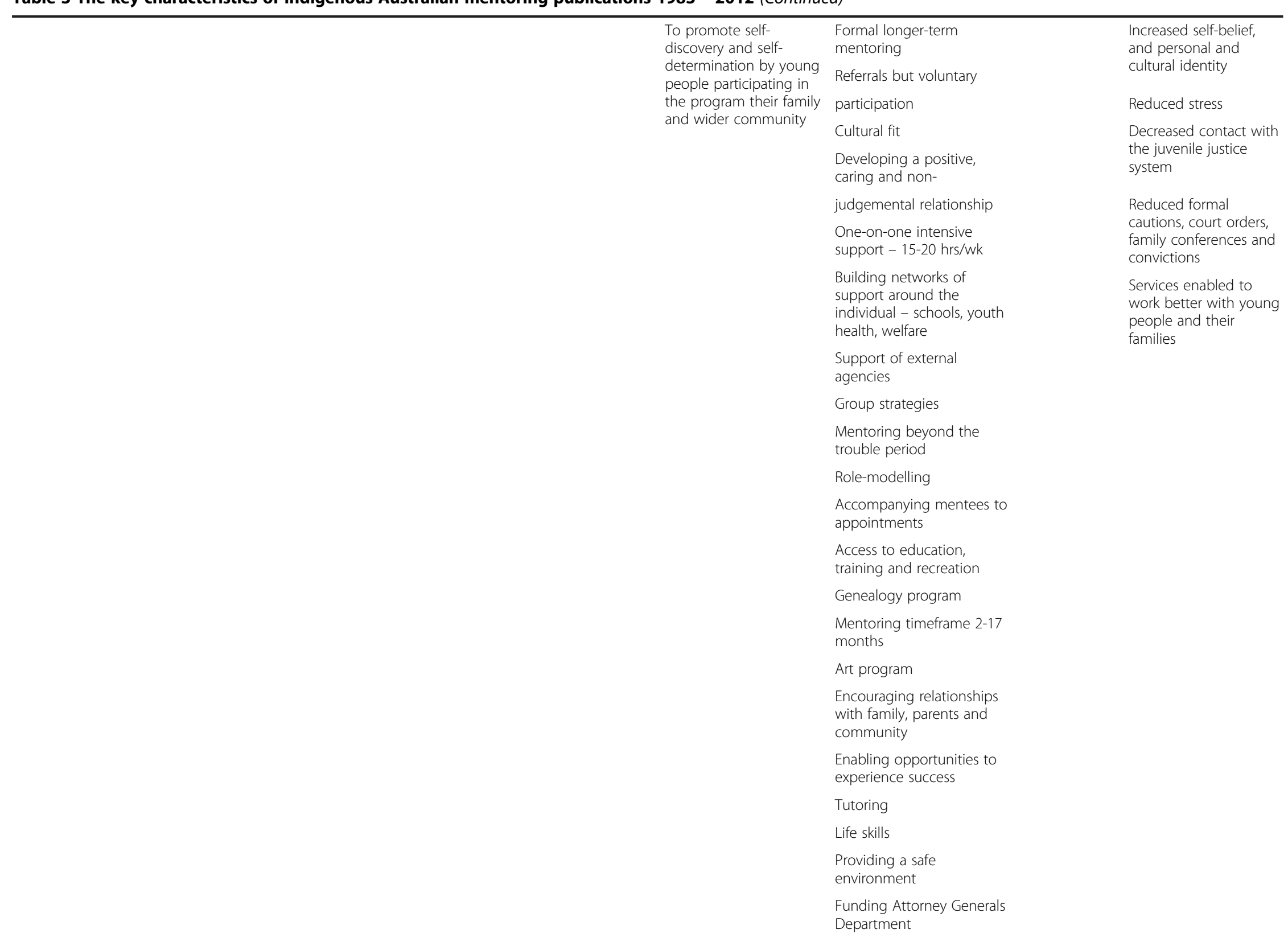


Table 3 The key characteristics of Indigenous Australian mentoring publications 1983 - 2012 (Continued)

\begin{tabular}{|c|c|c|c|c|c|c|c|c|}
\hline \multirow{16}{*}{$\begin{array}{l}\text { Brereton } \\
\text { \&Taufatofua, } 2010 \\
\text { Report }\end{array}$} & \multirow{16}{*}{$\begin{array}{l}\text { Indigenous } \\
\text { Australian } \\
\text { Mentoring } \\
\text { Programs in } \\
\text { employment }\end{array}$} & \multirow{16}{*}{$\begin{array}{l}\text { Workplaces } \\
\text { Australia- } \\
\text { wide }\end{array}$} & \multirow[t]{16}{*}{ Indigenous Australians } & \multirow[t]{16}{*}{ Not relevant } & \multirow{16}{*}{$\begin{array}{l}\text { Overview of Indigenous } \\
\text { mentoring programs } \\
\text { across Australia aimed at } \\
\text { increasing participation } \\
\text { in employment }\end{array}$} & Tailored to individual needs & \multirow{16}{*}{$\begin{array}{l}\text { Review } \\
\text { Descriptive }\end{array}$} & \multirow{16}{*}{$\begin{array}{l}\text { Only 'expected' } \\
\text { outcomes reported }\end{array}$} \\
\hline & & & & & & Flexibility of the workplace & & \\
\hline & & & & & & $\begin{array}{l}\text { Resources, training and } \\
\text { support for the mentor and } \\
\text { mentee }\end{array}$ & & \\
\hline & & & & & & $\begin{array}{l}\text { Cultural awareness training } \\
\text { for Indigenous and non- } \\
\text { Indigenous mentors }\end{array}$ & & \\
\hline & & & & & & Acknowledging difference & & \\
\hline & & & & & & $\begin{array}{l}\text { Incorporating and valuing } \\
\text { local knowledge }\end{array}$ & & \\
\hline & & & & & & Experiential learning & & \\
\hline & & & & & & $\begin{array}{l}\text { Formal evaluation for } \\
\text { program improvement }\end{array}$ & & \\
\hline & & & & & & Role-modelling & & \\
\hline & & & & & & $\begin{array}{l}\text { Links to support resources } \\
\text { and services }\end{array}$ & & \\
\hline & & & & & & $\begin{array}{l}\text { Appropriate empathy and } \\
\text { listening skills }\end{array}$ & & \\
\hline & & & & & & $\begin{array}{l}\text { Accountability and } \\
\text { reliability of mentors and } \\
\text { mentees }\end{array}$ & & \\
\hline & & & & & & $\begin{array}{l}\text { Developing rapport and } \\
\text { building trust }\end{array}$ & & \\
\hline & & & & & & $\begin{array}{l}\text { Setting and reaching goals } \\
\text { for the mentee }\end{array}$ & & \\
\hline & & & & & & $\begin{array}{l}\text { Bridging closure to the } \\
\text { relationship }\end{array}$ & & \\
\hline & & & & & & Funding not relevant & & \\
\hline \multirow{7}{*}{$\begin{array}{l}\text { Australian } \\
\text { Indigenous } \\
\text { Mentoring } \\
\text { Experience, } 2009 \\
\text { Report }\end{array}$} & \multirow{7}{*}{$\begin{array}{l}\text { Youth Mentoring } \\
\text { into Education }\end{array}$} & \multirow{2}{*}{$\begin{array}{l}\text { Australian } \\
\text { secondary } \\
\text { schools }\end{array}$} & \multirow{7}{*}{$\begin{array}{l}\text { Indigenous secondary } \\
\text { school students Years } \\
9-12\end{array}$} & \multirow{7}{*}{$\begin{array}{l}4 \text { universities; } \\
13 \text { staff; } 325 \\
\text { mentees; } 500 \\
\text { mentors; } 30 \\
\text { high schools }\end{array}$} & \multirow{3}{*}{$\begin{array}{l}\text { Increase Year } 10 \& 12 \\
\text { progression rates } \\
\text { Increase Year } 12 \text { to } \\
\text { university progression }\end{array}$} & One-on-one mentoring & \multirow{7}{*}{$\begin{array}{l}\text { Case Report } \\
\text { Descriptive }\end{array}$} & \multirow{7}{*}{$\begin{array}{l}\text { Increases across } \\
\text { progressions for Years } \\
\text { 9-10 (88\% AIME } \\
\text { compared to 81\% } \\
\text { National); Years 10-11 } \\
\text { (81\% AIME compared } \\
\text { to 59\% National); Years } \\
11-12 \text { (92\% AIME } \\
\text { compared to 63\% } \\
\text { National) } \\
\text { Increases Year 12 } \\
\text { completion (73\% AIME }\end{array}$} \\
\hline & & & & & & Voluntary participation & & \\
\hline & & \multirow{5}{*}{$\begin{array}{l}\text { East Coast of } \\
\text { Australia }\end{array}$} & & & & Voluntary mentors & & \\
\hline & & & & & \multirow[t]{4}{*}{$\begin{array}{l}\text { Work with } 6000 \\
\text { Indigenous secondary } \\
\text { school students by } 2020\end{array}$} & $\begin{array}{l}1 \text { hour/wk for } 17 \text { week } \\
\text { intensive program }\end{array}$ & & \\
\hline & & & & & & Learning Centres & & \\
\hline & & & & & & $\begin{array}{l}\text { Community \& University } \\
\text { Engagement }\end{array}$ & & \\
\hline & & & & & & Role-models & & \\
\hline
\end{tabular}




\begin{tabular}{|c|c|c|c|c|c|c|c|c|}
\hline & & & & & & Shared social activities & & $\begin{array}{l}\text { compared 60\% } \\
\text { National) }\end{array}$ \\
\hline & & & & & & $\begin{array}{l}\text { Funding partners } \\
\text { (Universities), philanthropic } \\
\text { organisations, in-kind } \\
\text { support, fund-raising }\end{array}$ & & \multirow[t]{2}{*}{$\begin{array}{l}\text { Increases Year } 12 \text { to } \\
\text { university (38\% AIME } \\
\text { compared to } 1.25 \% \\
\text { National) }\end{array}$} \\
\hline & & & & & & \multicolumn{2}{|l|}{$\begin{array}{l}\text { Linking in mentees family } \\
\text { and community }\end{array}$} & \\
\hline \multirow{12}{*}{$\begin{array}{l}\text { Australian } \\
\text { Indigenous } \\
\text { Mentoring } \\
\text { Experience, } 2010 \\
\text { Report }\end{array}$} & \multirow[t]{12}{*}{$\begin{array}{l}\text { Youth Mentoring } \\
\text { into Education }\end{array}$} & \multirow{12}{*}{$\begin{array}{l}\text { Australian } \\
\text { secondary } \\
\text { schools East } \\
\text { Coast of } \\
\text { Australia }\end{array}$} & \multirow{12}{*}{$\begin{array}{l}\text { Indigenous secondary } \\
\text { school students Years } \\
9-12\end{array}$} & \multirow[t]{12}{*}{$\begin{array}{l}7 \text { universities; } \\
388 \text { mentees }\end{array}$} & $\begin{array}{l}\text { Increase Year } 10 \& 12 \\
\text { progression rates }\end{array}$ & \multirow[t]{3}{*}{ One-on-one mentoring } & \multirow[t]{12}{*}{$\begin{array}{l}\text { Case Report } \\
\text { Descriptive }\end{array}$} & \multirow{7}{*}{$\begin{array}{l}\text { Increases across } \\
\text { progressions for Years } \\
9-10 \text { ( } 88 \% \text { AIME } \\
\text { compared to } 91.5 \% \\
\text { National); Years } 10-11 \\
\text { (87\% AlME compared } \\
\text { to } 74 \% \text { National); Years } \\
11-12 \text { (86\% AIME } \\
\text { compared to }\end{array}$} \\
\hline & & & & & $\begin{array}{l}\text { Increase Year } 12 \text { to } \\
\text { university progression }\end{array}$ & & & \\
\hline & & & & & $\begin{array}{l}\text { Work with } 6000 \\
\text { Indigenous secondary } \\
\text { school students by } 2020\end{array}$ & & & \\
\hline & & & & & & Voluntary participation & & \\
\hline & & & & & & Voluntary mentors & & \\
\hline & & & & & & 1 hour/wk for17 week & & \\
\hline & & & & & & intensive program & & \\
\hline & & & & & & Learning Centres & & $66.7 \%$ National) \\
\hline & & & & & & $\begin{array}{l}\text { Community \& University } \\
\text { Engagement }\end{array}$ & & $\begin{array}{l}\text { Increases Year } 12 \\
\text { completion }(100 \% \text { AIME } \\
\text { compared } 71.8 \%\end{array}$ \\
\hline & & & & & & Role-models & & National) \\
\hline & & & & & & Shared social activities & & Increases Year 12 to \\
\hline & & & & & & $\begin{array}{l}\text { Funding partners } \\
\text { (Universities), philanthropic } \\
\text { organisations, in-kind } \\
\text { support, fund-raising }\end{array}$ & & $\begin{array}{l}\text { university (38\% AlME } \\
\text { compared to } 10 \% \\
\text { National) }\end{array}$ \\
\hline Australian & Youth Mentoring & Australian & Indigenous secondary & & Increase Year $10 \& 12$ & One-on-one mentoring & Case Report & \\
\hline $\begin{array}{l}\text { Indigenous } \\
\text { Mentoring }\end{array}$ & into Education & $\begin{array}{l}\text { secondary } \\
\text { schools }\end{array}$ & $\begin{array}{l}\text { school students Years } \\
9-12\end{array}$ & $\begin{array}{l}\text { universities;30 } \\
\text { staff; } 566\end{array}$ & progression rates & Voluntary participation & & \\
\hline $\begin{array}{l}\text { Experience, } 2011 \\
\text { Report }\end{array}$ & & $\begin{array}{l}\text { East Coast of } \\
\text { Australia }\end{array}$ & & mentees & $\begin{array}{l}\text { Increase Year } 12 \text { to } \\
\text { university progression }\end{array}$ & Voluntary mentors & & \\
\hline & & & & & $\begin{array}{l}\text { Work with } 6000 \\
\text { Indigenous secondary }\end{array}$ & $\begin{array}{l}1 \text { hour/wk for } 17 \text { week } \\
\text { intensive program }\end{array}$ & & \\
\hline & & & & & school students by 2020 & Learning Centres & & \\
\hline & & & & & & $\begin{array}{l}\text { Community \& University } \\
\text { Engagement }\end{array}$ & & \\
\hline & & & & & & Role-models & & \\
\hline & & & & & & Shared social activities & & \\
\hline
\end{tabular}


Table 3 The key characteristics of Indigenous Australian mentoring publications 1983 - 2012 (Continued)

\begin{tabular}{|c|c|c|c|c|c|c|c|c|}
\hline & & & & & & $\begin{array}{l}\text { Funding partners } \\
\text { (Universities), philanthropic } \\
\text { organisations, in-kind } \\
\text { support, fund-raising }\end{array}$ & & \\
\hline \multirow[t]{10}{*}{$\begin{array}{l}\text { Department of Local } \\
\text { Government, } 1999 \\
\text { Report }\end{array}$} & \multirow[t]{10}{*}{$\begin{array}{l}\text { Aboriginal Political } \\
\text { Mentoring } \\
\text { Program }\end{array}$} & $\begin{array}{l}\text { Local } \\
\text { Councils }\end{array}$ & \multirow[t]{10}{*}{$\begin{array}{l}\text { Local Aboriginal } \\
\text { community members }\end{array}$} & \multirow[t]{10}{*}{$\begin{array}{l}27 \\
\text { participants }\end{array}$} & \multirow{10}{*}{$\begin{array}{l}\text { Encourage Aboriginal } \\
\text { people to run for local } \\
\text { elections } \\
\text { Educate the Aboriginal } \\
\text { community on the local } \\
\text { government process } \\
\text { and the importance of } \\
\text { Aboriginal input into } \\
\text { local government }\end{array}$} & \multirow{3}{*}{$\begin{array}{l}\text { Voluntary participation by } \\
\text { mentees but selection } \\
\text { process }\end{array}$} & $\begin{array}{l}\text { Original } \\
\text { Research }\end{array}$ & \multirow{3}{*}{$\begin{array}{l}\text { Not able to be } \\
\text { definitively assessed: } \\
\text { Increased political } \\
\text { participation - } 11 / 27 \text { ran } \\
\text { for election and } 2 \text { were } \\
\text { elected }\end{array}$} \\
\hline & & \multirow{9}{*}{$\begin{array}{l}\text { Kyogle Area } \\
\text { NSW }\end{array}$} & & & & & $\begin{array}{l}\text { Intervention } \\
\text { Research }\end{array}$ & \\
\hline & & & & & & & Strong & \\
\hline & & & & & & $\begin{array}{l}\text { Mentors - existing } \\
\text { relationships with mentee } \\
\text { and Aboriginal community; } \\
\text { understanding of Aboriginal } \\
\text { culture; vast experience of } \\
\text { local government and } \\
\text { supervision }\end{array}$ & & \multirow{4}{*}{$\begin{array}{l}\text { Increases across } \\
\text { progressions for Years } \\
9-10 \text { ( } 97 \% \text { AIME } \\
\text { compared to } 91.5 \% \\
\text { National); Years } 10-11 \\
(92.6 \% \text { AlME compared } \\
\text { to } 74 \% \text { National); Years } \\
11-12 \text { (79\%AIME } \\
\text { compared to } 66.7 \% \\
\text { National) } \\
\text { Increases Year 12 } \\
\text { completion (87.5\% } \\
\text { AlME compared } 71.8 \% \\
\text { National) }\end{array}$} \\
\hline & & & & & & Mutual matching & & \\
\hline & & & & & & \multirow{2}{*}{$\begin{array}{l}\text { One-on-one mentoring but } \\
\text { multiple mentors }\end{array}$} & & \\
\hline & & & & & & & & \\
\hline & & & & & & $\begin{array}{l}\text { Supported in political } \\
\text { activities and social support }\end{array}$ & & \multirow{3}{*}{$\begin{array}{l}\text { Increases Year } 12 \text { to } \\
\text { university ( } 35.7 \% \text { AIME } \\
\text { compared to } 10 \% \\
\text { National) }\end{array}$} \\
\hline & & & & & & 6 months + relationship & & \\
\hline & & & & & & $\begin{array}{l}\text { Funding Department of } \\
\text { Local Government NSW }\end{array}$ & & \\
\hline \multirow[t]{8}{*}{$\begin{array}{l}\text { PASS Australia } 2012 \\
\text { Web Page }\end{array}$} & \multirow{8}{*}{$\begin{array}{l}\text { Mentoring } \\
\text { Program for } \\
\text { Indigenous } \\
\text { students in years } \\
10,11 \text { and } 12\end{array}$} & \multirow{8}{*}{$\begin{array}{l}\text { Secondary } \\
\text { Schools } \\
\text { Queensland }\end{array}$} & \multirow[t]{8}{*}{$\begin{array}{l}\text { Indigenous secondary } \\
\text { school students }\end{array}$} & \multirow[t]{8}{*}{$\begin{array}{l}525 \text { students } \\
\text { in } 2011\end{array}$} & \multirow{8}{*}{$\begin{array}{l}\text { To improve the lives of } \\
\text { Indigenous Youth, } \\
\text { through Education, } \\
\text { Leadership and } \\
\text { Mentoring, by providing } \\
\text { them with the tools to } \\
\text { become strong } \\
\text { community leaders for } \\
\text { the future }\end{array}$} & $\begin{array}{l}\text { Group and one-on-one } \\
\text { mentoring }\end{array}$ & \multirow[t]{8}{*}{$\begin{array}{l}\text { Program } \\
\text { Description }\end{array}$} & Program graduations \\
\hline & & & & & & $\begin{array}{l}\text { Voluntary participation } \\
\text { (mentees) Paid mentors }\end{array}$ & & $\begin{array}{l}\text { Vocational qualification } \\
\text { - TAFE Certificates }\end{array}$ \\
\hline & & & & & & 1 day per week for 2 years & & \\
\hline & & & & & & $\begin{array}{l}\text { Indigenous and non- } \\
\text { Indigenous mentors }\end{array}$ & & \\
\hline & & & & & & Paid employment & & \\
\hline & & & & & & $\begin{array}{l}\text { Exposure to employment } \\
\text { pathways, community work } \\
\text { and further education }\end{array}$ & & \\
\hline & & & & & & $\begin{array}{l}\text { 6-9 Merit points towards } \\
\text { School Certificate }\end{array}$ & & \\
\hline & & & & & & Cultural program & & \\
\hline
\end{tabular}


Table 3 The key characteristics of Indigenous Australian mentoring publications 1983 - 2012 (Continued)

\begin{tabular}{|c|c|c|c|c|c|c|c|c|}
\hline \multirow{22}{*}{$\begin{array}{l}\text { New South Wales } \\
\text { Government, } 2010 \\
\text { Web Page }\end{array}$} & \multirow{22}{*}{$\begin{array}{l}\text { Workplace } \\
\text { Mentoring } \\
\text { Program for } \\
\text { Aboriginal people } \\
\text { working in the } \\
\text { NSW public sector }\end{array}$} & \multirow{22}{*}{$\begin{array}{l}\text { Public Sector } \\
\text { NSW }\end{array}$} & \multirow{22}{*}{$\begin{array}{l}\text { Aboriginal people } \\
\text { working in the NSW } \\
\text { public sector }\end{array}$} & & \multirow{22}{*}{$\begin{array}{l}\text { Mentoring guidelines to } \\
\text { improve employment, } \\
\text { training and career } \\
\text { development } \\
\text { opportunities for } \\
\text { Aboriginal people in the } \\
\text { NSW public sector }\end{array}$} & Sports program & & \\
\hline & & & & & & Career Pathways Program & & \\
\hline & & & & & & Leadership & & \\
\hline & & & & & & $\begin{array}{l}\text { Education to employment } \\
\text { transitioning }\end{array}$ & & \\
\hline & & & & & & Industry work experience & & \\
\hline & & & & & & $\begin{array}{l}\text { Industry, community and } \\
\text { school partners }\end{array}$ & & \\
\hline & & & & & & Awards Celebrations & & \\
\hline & & & & & & $\begin{array}{l}\text { Funding Department of } \\
\text { Education, Employment \& } \\
\text { Workplace Relations }\end{array}$ & & \\
\hline & & & & \multirow[t]{14}{*}{ Not relevant } & & $\begin{array}{l}\text { Modelling desirable } \\
\text { behaviours and attitudes }\end{array}$ & \multirow[t]{14}{*}{$\begin{array}{l}\text { Program } \\
\text { Description }\end{array}$} & \multirow[t]{14}{*}{ Not relevant } \\
\hline & & & & & & One-on-one mentoring & & \\
\hline & & & & & & $\begin{array}{l}\text { Helping the mentee } \\
\text { understand the values of } \\
\text { the agency }\end{array}$ & & \\
\hline & & & & & & $\begin{array}{l}\text { Actively listening to the } \\
\text { mentee }\end{array}$ & & \\
\hline & & & & & & $\begin{array}{l}\text { Sharing your own relevant } \\
\text { stories and experiences }\end{array}$ & & \\
\hline & & & & & & $\begin{array}{l}\text { Helping the mentee to } \\
\text { identify their goals }\end{array}$ & & \\
\hline & & & & & & $\begin{array}{l}\text { Providing opportunities for } \\
\text { learning and reflection }\end{array}$ & & \\
\hline & & & & & & $\begin{array}{l}\text { Understanding of cultural } \\
\text { obligations }\end{array}$ & & \\
\hline & & & & & & $\begin{array}{l}\text { Encouraging the mentee to } \\
\text { develop new skills }\end{array}$ & & \\
\hline & & & & & & Offering career advice & & \\
\hline & & & & & & $\begin{array}{l}\text { Guiding the mentee to } \\
\text { achieve objectives }\end{array}$ & & \\
\hline & & & & & & $\begin{array}{l}\text { Providing insights into the } \\
\text { culture of the agency }\end{array}$ & & \\
\hline & & & & & & $\begin{array}{l}\text { Offering constructive } \\
\text { feedback; and providing } \\
\text { regular encouragement }\end{array}$ & & \\
\hline & & & & & & Funding NSW Government & & \\
\hline
\end{tabular}


Table 3 The key characteristics of Indigenous Australian mentoring publications 1983 - 2012 (Continued)

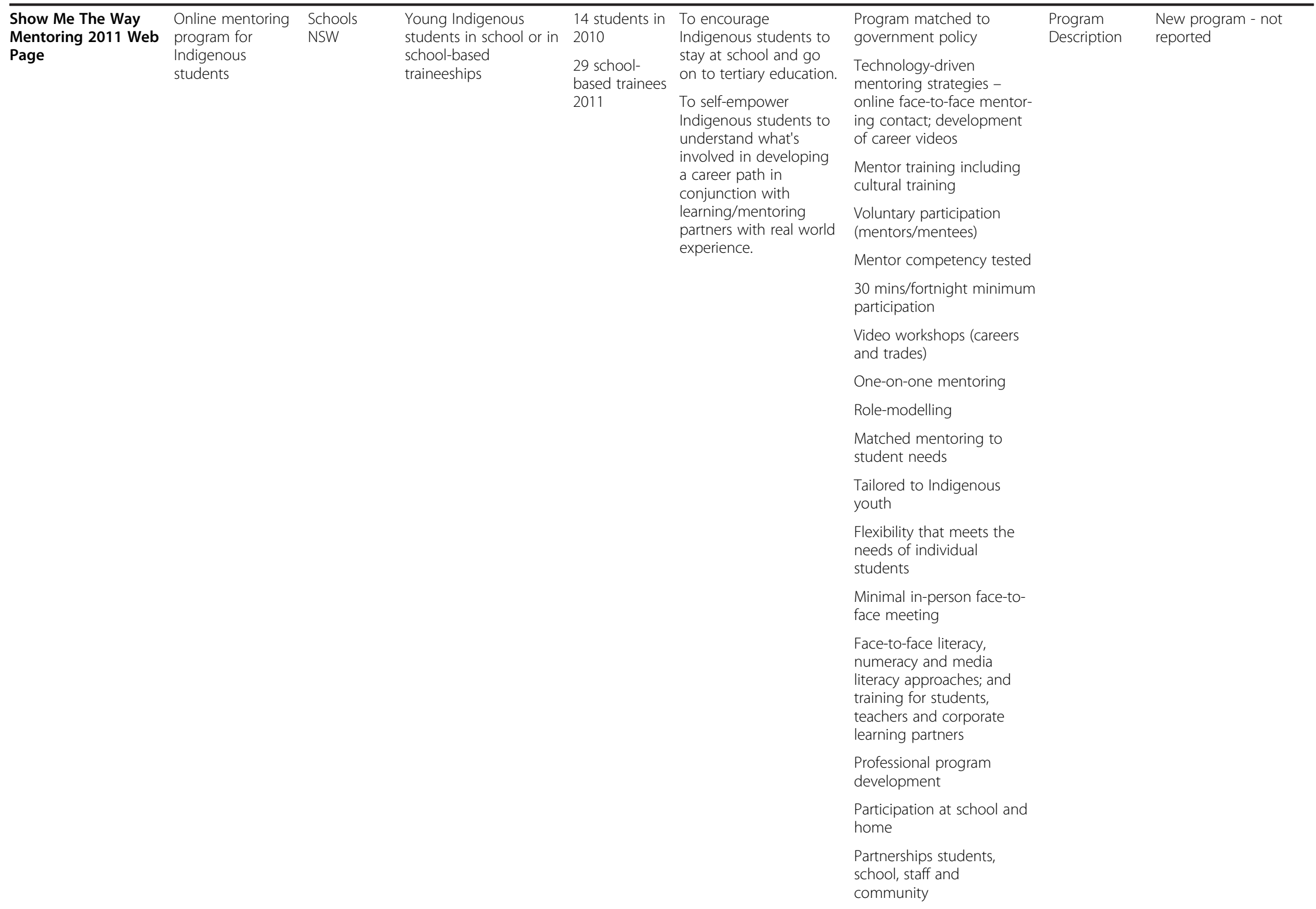


engagement

Implemented discretely,

and in conjunction with

existing school programs

Continuous quality

improvement strategies

embedded in the program

Reward system for

participation

Funding - charitable

organisation and corporate

partners 


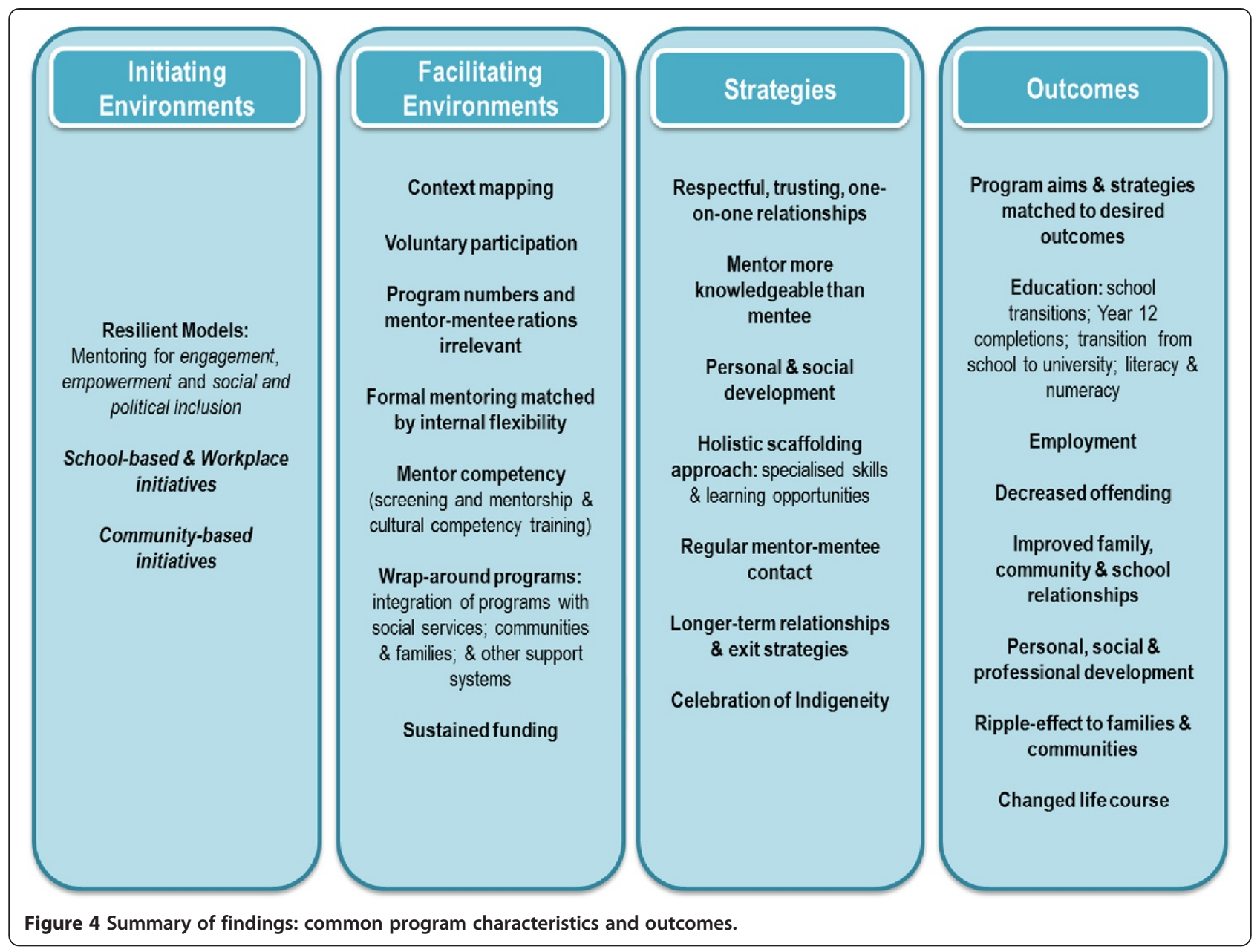

not report on this aspect of mentoring. The value of supporting the welfare of mentors registered in only 2/15 (13.3\%) publications; a lack of support for mentors was however, critiqued by Nasir [32].

Five/15 (33.3\%) publications explicitly demonstrated a reconciliatory approach other than partaking in cultural awareness training. For example, in the case of implementing a health promotion program, Paase and Adams [25] clearly embedded Indigenous protocols in their ways of working in the development of a peer mentoring program. This was evidenced by their reported consultations, valuing of local knowledge, cultural tailoring and delivery of the program. A high premium was also placed on the integration of mentoring with social services, communities and families and other support systems such as welfare agencies, corporate and industry partners, police, schools, health services, universities and training and recreation services; this was evidenced in 13/15 (\%) publications [23,25-29,31,33-36].

Resourcing for mentoring programs reported (13/15 or $86.6 \%$ ) was weighted in favour of government-derived funding with $7 / 13$ or $53.8 \%$ [23,24,26,28,30,31,33,37] identifying various government departments as their supporting body. Other funding sources ranged from charitable organisations that had the support of corporate partners [27] and programs which were dependent on partner organisations, philanthropic investments, fundraising and in-kind support [34-36] to workplace backing $[29,32]$.

\section{Strategies}

Mentoring strategies are the mechanisms that underlie mentoring relationships. While publications contributing to the review described diverse and sometimes poorly delineated processes in mentoring programs, four common program elements were evident in the mentoring models. These strategies were: 1) developing respectful, trusting, one-on-one mentoring relationships; 2) promoting personal and social development; 3) integrating social services, organisations and other support systems including communities and families; and 4) increasing specialised skills.

Developing respectful, trusting, one-on-one mentoring relationships was reported in $13 / 15$ or $86.6 \%$ of cases 
[23,25-29,31-37]. In the two other cases, PASS Australia [26] used group and one-on-one mentoring, while the health promotion program [25] used peer group and oneon-one mentoring practices. Seven/15 publications reported timeframes: School programs AIME [34-36] and National Indigenous Mentoring Pilots Program [31] invested one hour per week; Show Me The Way [27] a minimum of 30 minutes fortnightly; PASS Australia [26] one day per week; while the Panyappi Program [33] reported an intensive 15-20 hours weekly with young people. Strategies used to create trusting relationships between mentors and mentees included aiming for longerterm relationships; social/shared activities such as fishing, gardening, dance, sports, art, chess and camps and group activities; strategies that promoted friendships such as mentors sharing personal stories, being reliable and accountable, and providing constructive feedback and regular encouragement; demonstrated acceptance of mentees including empathy, non-judgemental attitudes, caring, humour, listening and bridging closure to relationships; mentors walking through life with mentees; and cultural, gender and interest matching.

Promoting personal and social development featured as a common and necessarily important strategy occurring in programs and mentor-mentee interactions. This saturation occurred with the exception of Nasir [32]. Nasir criticised mentoring practices in an organisation that aimed to improve the recruitment, retention and completion rates of Indigenous Australian apprentices. She argued that mentoring in this instance was one-way and neglected to take account of Indigenous Australian ways of knowing, being and doing in practice. Fourteen/15 or 93.3\% of publications explicitly incorporated acknowledging Indigeneity as a strategy for promoting personal and social development for mentees. There was acknowledgement of the centrality of cultural mores for Indigenous Australian people to attend to the complexity of cross-cultural situations and acknowledgement of historical power relations. Recognised across publications was that when groups of Indigenous Australians gather together, there is important cultural work conducted in terms of sharing and making meaning of Indigeneity. Driving strategies that acknowledged Indigeneity were recognising Indigenous ways of knowing, being and doing by having the consistent endorsement of, and valuing of Elder/family/community-inclusive relationships and knowledge. Programs were thus culturally-tailored to mentee needs. Various other approaches used to translate this strategy into practice ranged from having Indigenous Australian reference groups guiding mentoring processes, through having the flexibility in program implementation to account for cultural protocols and differences, incorporating genealogy programs to connect with identity building work, and partnering with local Indigenous
Australian organisations, to engaging Indigenous Australian role-models as the faces of programs.

Most publications, 12/15 (80\%) described integrated mentoring models - implementing a circle of care and support around mentees to support personal and social development. The three cases in which outside support was not integrated were all workplace mentoring programs [24,30,32]: Nasir [32] critiqued the program supporting apprentices for this absence. This holistic scaffolding approach meant that programs built-in networks of support and resources around mentees as part of their mentoring practices. Stacey [33] demonstrated this well in her evaluation of Panyappi, a youth mentoring program. It aimed first to intervene in pathways of offending behaviour and second, to decrease young participants' contact with the juvenile justice system and/or agencies associated with this system. A third aim sought to promote self-discovery and self-determination by young people participating in the program, their families and wider community. Stacey reported that Panyappi worked in conjunction with a range of health, family, education and community services. The integrated model also had mutual benefits for the program and services; it enabled services to work better with young people and their families and referrals were received from these services to Panyappi. This was also an outcome of the mentoring relationship. In addition to the previouslymentioned support structures, other wrap-around services in integrated models included psychological support and counselling. These strategies served to strengthen social and cultural capital; this allegedly worked both ways in mentoring relationships - for mentees and mentors.

All publications focussed to varying degrees on increasing specialised skills for mentees, and to a lesser extent building mentor skills. Strategies varied according to the nature of the mentoring program (its goals, objectives and the backgrounds of mentees and mentors) and the existing skill sets within that cohort. Matching appropriate skills and development to the program aims were crucial for the overall success of programs and was resourced as much as possible through the mentors. For example, the AIME program [34-36] had academic achievement and university enrolment as goals and thus situated the program in schools, incorporated Learning Centres into their program and co-ordinated with local universities to recruit their mentors.

Increasing specialised skills for mentees and mentors also involved accessing the cadre of organisations, services and/or other human resources that were integrated into the programs to support skill development. Targeted skills sets for mentees focussed on learning opportunities including literacy and numeracy; career development skills such as resume writing and work ethic and work experience; areas in the achievement of mentee goals such as education in the political system; and vocationally- 
oriented curriculum (building and construction, art, horticulture, hospitality and maritime). For mentors, increased skills most often took the form of cultural competency training and professional development through mentoring courses.

\section{Outcomes}

Not all publications recounted the extent to which programs were effective. Of the nine that did, the rationales for program development intimated that a range of outcomes was being targeted. To a large extent, outcomes were matched to program aims. At times however, consequences of programs were unexpected. Most outcomes were qualitatively investigated and described, with only three reports quantitatively measuring their achievements [34-36]. AIME provides an excellent example of the aforementioned. The goals of the AIME Program for 2009, 2010, 2011 were to work with Indigenous Australian secondary students to improve student progressions between Years 9, 10, 11 and 12; increase the number of Year 12 completions; and grow the numbers of students transitioning from Year 12 to university. Thus measures reflected these goals and showed significant changes. In 2009, 2010 and 2011 respectively, and corresponding to AIME's targets, reported were increases across progressions for Years 9-10 (88\%, 88\%, 97\% AIME compared to $81 \%, 91.5 \%, 91.5 \%$ Indigenous students Nationally); Years $10-11$ (81\%, 87\%, 92.6\% AIME compared to 59\%, $74 \%, 74 \%$ of Indigenous students Nationally); and Years 11-12 (92\%, 86\%, 79\% AIME compared to 63\%, 66.7\%, $66.7 \%$ Indigenous students Nationally). There was also increases for Year 12 completions (73\%, 100\%, 87.5\% AIME compared 60\%, 71.8\%, 71.8\% Indigenous students Nationally); and increases for Year 12 to university (38\%, 38\%, 35.7\% AIME compared to $1.25 \%, 10 \%, 10 \%$ Indigenous students Nationally).

Likewise the Tribal Warriors program [28] for postrelease Indigenous Australian men described decreased re-offending, including a decrease of $80 \%$ in men charged with robbery; and improved relationships between police and the Aboriginal community. Dawes \& Dawes [23] also commented on positive relationships as the outcome of their program for young people in a detention centre. In the Panyappi mentoring program for young people who had, or were at risk of offending behaviours, Stacey [33] qualitatively reported: marked changes in offending behaviour; decreased frequency of offending; decreased contact with the juvenile justice system; and reduced formal cautions, court orders, family conferences and convictions. Other outcomes that corresponded with program aims included attitude shifts; increased self-belief, and personal and cultural identity; reduced stress; and enhanced capacity of services to work better with young people and their families.
In school environments, MacCallum, Beltman \& Palmer (2005) [31] found that mentees experienced increased self-confidence and self-esteem; school attendance; retention; and participation in classroom tasks; enhanced valuing of school and connections between school and work; increased ability to solve personal and social problems; development of leadership and life skills; improved relationships with, and between peers, teachers and family members; and improved literacy and numeracy. They also revealed that mentors showed improved knowledge of Indigenous Australian culture and youth issues; development of strong relationships with students; and enhanced personal development and self-esteem. The flow-on effects of the mentoring program impacted beyond the central mentoring relationships to the school and community: enhanced links between school and community; increased involved of families in school; awareness of, and access to local Indigenous role-models; development of inter-school relationships; and positive contact between Indigenous and non-Indigenous Australian families. Similarly, in the university setting, Burgess and Dyer [29] showed that of 12/20 program completions, 8 gained internal university employment; and four found external university positions. The health promotion mentoring program [25] identified smoking cessation, skill acquisition, increased expression of identity, and a reduction in cultural isolation as program outcomes.

\section{Discussion}

\section{Quantity, nature and quality}

The findings show that literature around Indigenous Australian mentoring has an emergent lifespan of 14 years. They revealed that while outputs have increased, the volume of original research publications has not increased across time. All original research outputs were qualitatively-oriented. The methodological adequacy of the identified original research publications was strong; however, only one was peer-reviewed. There was no pattern in the volume of original research publications; these were randomly dispersed in number and nature across a 10 year lifespan from 1999 - 2009. This is not surprising given that publication outputs were heavily weighted in favour of descriptive documents: fourteen/ 15 publications were labelled descriptive and only one as intervention research. Specifically, such an output record means that Indigenous Australian mentoring research is still in its early exploratory phase and little research has explored the effectiveness of mentoring in its various forms, captured its impact qualitatively or quantitatively, developed appropriate measures or assessed it cost-effectiveness. This pattern is consistent with the results of several other reviews across a number of Indigenousspecific areas, including health, program transfer and implementation, sexual assault, cultural competency, child 
and maternal health and suicide prevention [10-15]. The stop-start nature of Indigenous funding patterns, lacking consideration for the inclusion of evaluative components in program implementation and short-term research funding all slow contributions toward the productive trend of more rigorous strategic intervention research $[10,38]$. While qualitative research is valuable in setting a baseline, it might "not maximise research benefits" [10]. Thus more compelling advances in the field of mentoring require paradigm-shifting approaches. Despite the concentration of included publications on describing the elements and outcomes of Indigenous Australian mentoring experiences ${ }^{\mathrm{b}}$ however, these can provide valuable evidence and lessons for developing the field further. The slow progression of Indigenous Australian mentoring research is consistent with the findings of others in the broader field of mentoring. For instance, Crisp \& Cruz [39] noted that "mentoring research has made little progress in identifying and implementing a consistent definition and conceptualization of mentoring, is largely atheoretical and is lacking in terms of rigorous quantitative research designs that allow for testing the external validity of findings".

\section{Mentoring characteristics}

The main characteristics of Indigenous Australian mentoring were summarised in Figure 4. The key components of Indigenous Australian mentoring aligned quite closely with non-Indigenous and international theorising on mentoring. These theorists suggest that mentoring work, as also evidenced in this review, must fit the needs and characteristics of the target group. Ideally, program elements should work in synergy, but those deemed most important and supported by this review are: duration (length of the relationship); intensity (frequency of contact); extent of integration with other services; programs matched to target groups (high-risk populations vis-à-vis high-performance populations); a focus on approaches (formal or informal and cultural appropriateness) [7,40-43]; sustainable funding arrangements; and community buy-in [44]. The key differences found in this review were the ways programs accounted for and celebrated cultural differences in their implementation. This difference meant strategies such as training in cultural competencies became important; along with screening mentors for individual characteristics and attitudes such as respect and acceptance. However, to be noted is that there is limited evidence to suggest a lack of fitness between overseas mentoring models and local contexts [5]. In fact, DuBois et al. [4] stated that the practice of matching on race, gender and interests does not impact program effectiveness to the degree initially thought.

This review included a range of mentoring initiatives aimed at increasing Indigenous Australian empowerment, engagement and social inclusion across a number of different developmental areas; particularly in education (school-based initiatives), employment (workplace initiatives) and broader health and wellbeing. Initiatives were embedded in ecological models of resilience models that worked from the premise of providing opportunities for building on existing strengths for mentees, mentors, families and communities, and service providers. In individual programs, mentee numbers and mentor-mentee ratios varied and appeared irrelevant; that is, outcomes were equally recorded against programs regardless of the participant intake or mentees allocated to each mentor. All publications predominately described a clear orientation toward formal mentoring processes, meaning that while participation for mentors and mentees was voluntary and there was internal flexibility, there were certain nonnegotiable aspects and formal organisation of programs. Generally however, there were a number of flexible elements that contributed to their success. Thus developed were innovative integrated models of mentoring programs with wrap-around social services, communities and families and other support systems. These models had a variety of educational and life skill-related supports and in some instances clinical support e.g. psychologists. Consequently, these models link mentees and mentors, and also connect many individuals and resources in the mentors' lives to the networks in the lives of the mentees. Since both mentees and mentors are connected to the program staff and service providers this approach has important implications for providing circles of holistic care needed to help programs deliver more targeted mentoring. To engage in this type of social mapping, it was evidenced that mentoring programs need to have a clear understanding of the environment and socio-cultural and economic milieu in which potential participants operate as well as clearly defined aims and outcomes to make a good match with strategies. How these criteria manifest, informs the type of program and its operational strategies.

In order to enhance the benefits of mentoring, a focus on mentees' support and development was matched with training and development of mentors. Mentor competency, particularly training in mentorship and cultural awareness were evidenced as critical prerequisites for successful program implementation. Given the long-term nature of successful mentoring practices, sustained funding was critically noted as vital to maximising the benefits of mentoring.

Mentoring strategies were manifest in many different forms. As previously noted, mentoring was often reported as one strategy of a more extensive support/development program. Programs were culturally-tailored to meet mentee needs, both through implementing the integrated model approach (inclusion of Elders, families and communities) and acknowledging Indigeneity by ensuring 
cross-cultural training was provided to mentors. But support for Indigenous Australians through mentoring, like most other Indigenous programs, has often used short-term approaches. This approach is contrary to the evidence found in this review that longer-term investment is needed. The call for longer-term mentoring is made in international mentoring literature. Indeed, this evidence that suggests inadequate implementation and short-term programs has potentially harmful effects on mentees $[6,45]$. This review showed that mentoring impacts most positively within a respectful longer-term mentoring relationship characterised by trust and regular one-on-one mentor contact. The amount of contact time in the mentoring relationship was flexible; however, the more vulnerable the mentee the more contact time needed was reported. Strategies also featured opportunities for reciprocity between mentees and mentors. However, mentors were considered to be more knowledgeable, particularly in the field in which the program developed. One important but not necessarily intended outcome of such engagement was that whether or not programs committed to a reconciliatory approach, respect for Indigenous Australian cultures and reconciliatory attitudes were consequences of mentoring processes in a number of cases.

Personal and social development of the mentee was supported by a holistic scaffolding approach in which specialised skills and learning opportunities were provided. These development opportunities also extended to mentors. Mentors were provided with training in mentorship to ensure they had knowledge of and exhibited the most engaging mentor characteristics.

The ways mentoring was used to support Indigenous Australians looked very different across program aims, and therefore a diverse number of outcomes were cited. Significant outcomes were realised in the largest proportion of mentoring publications, with clear benefits to both mentees and mentors, and others: with the exception of AIME reports [34-36], these benefits were not quantified. The nature of outcomes for Indigenous Australians however, matched the wide range of positive outcomes that are evidenced in other mentoring programs; for instance, behavioural, attitudinal, health including social and emotional wellbeing, interpersonal, motivational, and academic outcomes [4-6]. According to the broader non-Indigenous literature, mentoring benefits are more apparent for those facing complex environmental risks [42]; thus mentoring represents a good strategic investment for Indigenous people who have diverse needs. Because the mentoring process involves a reciprocal relationship in which all parties assume active roles, it needs to be identified as an investment in community development as well as the individual development of mentees.

\section{Limitations}

A rigorous search strategy was used and care taken to canvass a broad spectrum of multi-disciplinary peerreviewed and grey literature. However, there are always possibilities of oversight when searching publications and thus potential that all relevant studies were not located. For instance, the omission of the analogous search terms might have meant that we missed relevant mentoring studies subsumed under different terminology; and search facilities in individual databases differed. Relevant publications might have been misclassified or excluded. Nevertheless, elevated levels of agreement between blinded coders suggest relevant publications have been classified or excluded with high level accuracy. Timeframes canvassed began from the emergence of the earliest mentoring literature and thus allowed for the optimal extraction of patterns of publication quantities and methodological changes. A careful hand-searching of included publications was implemented to mine for relevant data on mentoring characteristics. However, the human component of implementation means it is possible some elements were not located.

\section{Conclusion}

The primary outcome of the review indicates that there is currently insufficient evidence from published documents to confidently prescribe a best practice model for Indigenous Australian mentoring. Nevertheless, there was sufficient frequency of qualitative reporting between the authors of publications to conclude that mentoring is potentially a valuable empowerment strategy, particularly in the areas of health and wellbeing, education and employment and as a remedial and preventative measure in reducing offending behaviours.

There is a lack of evidence from peer-reviewed publications or in the grey literature on the most effective strategies for mentoring Indigenous Australians. The only measurable outcomes for effective mentoring practices lies in mentoring programs for Indigenous Australian secondary students [34-36]. Importantly, the review of mentoring programs shows that there are different forms of mentoring that offer considerable promise for increasing participation rates in education and employment and in improving wellbeing (including health benefits, more active citizenry and socio-cultural inclusion and decreased risk of offending) for Indigenous Australians. An evidence-informed mentoring model would take into account the key findings of the review. There has been unquestioning implementation of mentoring programs. The review creates the rationale and need for providing formal mentoring programs with internal flexibility, such that mentoring strategies are tailored in collaboration with targeted Indigenous Australian communities to address their needs and preferences. Such tailoring is likely 
to be crucial for optimising acceptability and feasibility of program delivery as well as outcomes. Longer-term investments in evolving integrated models of mentoring with wrap-services (including families, communities, social services and other support systems) are germane to the development of effective mentoring practices for Indigenous populations in Australia. Key elements to consider for inclusion in such models are formal mentoring programs with internal flexibility; context-mapping; culturally-tailored programs; voluntary participation; mentor competencies (including both mentor training and cultural competence); regular one-on-one mentor contact; personal and social development; specialised skills and learning opportunities; long-term mentoring relationships; exit strategies; and celebrating Indigeneity within the program.

Methodologically, the review showed a predominance of descriptive publications in the field of Indigenous Australian mentoring across time. Descriptive research does not provide quality improvement in programs, nor does it provide evidence of how to improve programs or whether those programs were effective. There must be change in the patterns of research if the field is to advance. There is a real need to evaluate programs particularly in terms of outcomes and, given there were no economic evaluations, costs. Commitments to improving funding structures and attitudes toward research must change to improve mentoring outcomes across a range of social determinants of health for Indigenous Australians. While this review undertook to study mentoring for Indigenous Australians only, it is possible for the findings to be extrapolated to other similar structures, settings and populations.

\section{Endnotes}

${ }^{a}$ Empowerment is defined in this paper as: "a social action process by which individuals, communities, and organizations gain mastery over their lives in the context of changing their social and political environment to improve equity and quality of life" [46].

${ }^{\mathrm{b}}$ It should be noted that the three AIME [34-36] reports did cite specific measurable impacts but did not meet criteria to be classified as original research.

\section{Competing interests}

The authors declare that they have no competing interests.

\section{Authors' contributions}

$\mathrm{RB}, \mathrm{KT}, \mathrm{JM}$ and ST conceived of the study and participated in its design and coordination. RB took the lead role in searching, classifying and reviewing the literature, establishing the quality of intervention publications and drafted the paper. RB and ST collaborated on the classification and inclusion of publications. KT and JM edited the draft paper. All authors read and approved the final manuscript.

\section{Acknowledgments}

We acknowledge direction and financial support for this review from the Department of Aboriginal and Torres Strait Islander Affairs and the Australian Research Council.

\section{Author details}

${ }^{1}$ The Cairns Institute, James Cook University, PO Box 6811, Cairns 4870, Australia. ${ }^{2}$ Gunggandji Aboriginal Corporation, 1 Loban Lane, Yarrabah 4871 Australia.

Received: 3 September 2013 Accepted: 4 December 2014

Published: 13 December 2014

\section{References}

1. Wallerstein $\mathrm{N}$ : What is the evidence on effectiveness of empowerment to improve health? [http://www.euro.who.int/_data/assets/pdf_file/0010/ 74656/E88086.pdf]

2. Tsey K, Whiteside M, Haswell-Elkins M, Bainbridge R, Cadet-James Y, Wilson A: Empowerment and Indigenous Australian health: a synthesis of findings from Family Wellbeing formative research. Health Soc Care Community 2009, 18:169-179.

3. Israelashvili M, Wegman-Rozi O: Mentoring at-risk preschoolers: Lessons from the A.R.Y.A. Project. J Prim Prev 2005, 26(2):189-201.

4. DuBois DL, Karcher MJ: Handbook of youth mentoring. Thousand Oaks, Calif: SAGE; 2005.

5. Farruggia SP, Bullen P, Solomon F, Collins E, Dunphy A: Examining the cultural context of youth mentoring: A systematic review. J Prim Prev 2011, 32(5-6):237-251.

6. Jekielek S, Moore KA, Hair EC, Scarupa HJ: Mentoring: A promising strategy for youth development. Washington, D.C: Child Trends; 2002.

7. Moodie ML, Fisher J: Are youth mentoring programs good value-formoney? An evaluation of the Big Brothers Big Sisters Melbourne Program. BioMed Central Public Health 2009, 9(1):41.

8. Bozeman B, Feeney MK: Toward a useful theory of mentoring: A conceptual analysis and critique. Adminis Society 2007, 39(6):719-739.

9. Herceg A: Improving health in Aboriginal and Torres Strait Islander mothers, babies and young children: a literature review. Canberra: Commonwealth of Australia; 2005

10. Sanson-Fisher RW, Campbell EM, Perkins JJ, Blunden SV, Davis BB: Indigenous health research: a critical review of outputs over time. Australasian Med Publis Company 2006, 184(10):502-505.

11. McCalman J, Tsey K, Clifford A, Earles W, Shakeshaft A, Bainbridge R: Applying what works: a systematic review of the transfer and implementation of promising services and programs. BMC Public Health 2012, 12:600.

12. McCalman J, Bainbridge $R$, Clifford $A$, Tsey $K$ : A systematic literature search of cultural competence indicators and interventions targeting Indigenous peoples in Australia. New Zealand, United States and Canada: Sax Institute for the Mental Health Drug and Alcohol Office, NSW Ministry of Health; 2013.

13. McCalman J, Bridge F, Whiteside M, Bainbridge R, Tsey K, Jongen C: Responding to Indigenous Australian Sexual Assault. SAGE Open 2014, 4(1).

14. Jongen C, McCalman J, Bainbridge R, Tsey K: Aboriginal and Torres Strait Islander maternal and child health and wellbeing: a systematic search of programs and services in Australian primary health care settings. BMC Pregnancy and Childbirth 2014, 14(1):251.

15. Clifford A, Doran C, Tsey K: A systematic review of suicide prevention interventions targeting Indigenous peoples in Australia, United States, Canada and New Zealand. BMC Public Health 2013, 13:463.

16. Kram KE: Mentoring at work: Developmental relationships in organizational life. Glenview, IL: Scott, Foresman; 1985.

17. Bozeman B, Feeney MK: Toward a useful theory of mentoring: A conceptual analysis and critique. Admin Society 2007, 39(6):719-739.

18. Tolan P, Henry D, Schoeny M, Bass A: Mentoring interventions to affect juvenile delinquency and associated problems. [http://www.campbellcollaboration.org/ news_Promising_results_mentoring_programs.php]

19. Haggard D, Dougherty T, Turban D, Wilbanks J: Who is a Mentor? A review of evolving definitions and implications for research. J Manag 2011 37(1):280-304

20. Starcevich M: Coach, mentor: Is there a difference? [http://www. coachingandmentoring.com/Articles/mentoring.html] 
21. Critical Appraisal Skills Programme: Making sense of evidence about clinical effectiveness: Qualitative research checklist. [http://media.wix. com/ugd/dded87_951541699e9edc71ce66c9bac4734c69.pdf]

22. Preferred Reporting Items for Systematic Reviews and Meta-Analyses: PRISMA Flow Diagram. [http://www.prisma-statement.org/2.1.4\%20-\% 20PRISMA\%20Flow\%202009\%20Diagram.pdf]

23. Dawes G, Dawes C: Mentoring 2: A program for 'at risk' Indigenous youth. Youth Studies Australia 2005 2005, 24(4):45-49.

24. New South Wales Government: Aboriginal Mentoring Program: Guidelines for mentors. [http://www.miob.nsw.gov.au/htmldocs/guidelines-formentors/]

25. Paase $\mathrm{G}$, Adams $\mathrm{K}$ : Working together as a catalyst for change: The development of a peer mentoring model for the prevention of chronic disease in Australian Indigenous communities. Australian J Primary Health 2011, 17(3):214-219.

26. PASS Australia: PASS Australia: Education, leadership. Mentoring. [http://www.passaustralia.com.au/?page_id=27]

27. Show Me The Way Incorporated: Show Me The Way Business Plan. [http://www.showmetheway.org.au/SMTW\%20Business\%20Plan.pdf]

28. Warriors T: Mentoring Indigenous young people: the tribal warrior program. Judicial Officer Bullet 2011, 23:5-6.

29. Burgess J, Dyer S: Workplace mentoring for indigenous Australians: a case study. Equal Oppor Int 2009, 28(6):465-485.

30. Department of Local Government: Evaluation of Aboriginal Mentoring Program: Report. Sydney: New South Wales Government; 1999.

31. MacCallum J, Beltman S, Palmer D: The Australian Association for Research in Education Conference. Sydney: The Australian Association for Research in Education; 2005.

32. Nasir T: Two-way mentoring for Indigenous apprentices and staff. [http://www.avetra.org.au/AVETRA\%20WORK\%2011.04.08/CS5.2\%20-\% 20Tanyah\%20Nasir.pdf]

33. Stacey K: Panyappi Indigenous Youth Mentoring Program: External evaluation report. Adelaide: Metropolitan Aboriginal Youth Team, SA Department of Human Services; 2004.

34. Australian Indigenous Mentoring Experience Corporation: AIME Annual Report 2009. Redfern NSW: Australian Indigenous Mentoring Experience Corporation; 2009.

35. Australian Indigenous Mentoring Experience Corporation: AIME Annual Report 2010. Redfern, NSW: Australian Indigenous Mentoring Experience Corporation; 2010

36. Australian Indigenous Mentoring Experience Corporation: AIME Annual Report 2011. Redfern, NSW: Australian Indigenous Mentoring Experience Corporation; 2011

37. Brereton D, Taufatofua R: Good Practice in Mentoring of Indigenous Employees. Brisbane: Centre for Social Responsibility in Mining. University of Queensland; 2010

38. Bainbridge R, Tsey K, Andrews R, McCalman J, Brown C: Managing top-down change with bottom-up leadership: developing a community social and emotional wellbeing action framework in Aboriginal Australia. J Australian Indigenous Issues 2013, 16(2):20.

39. Crisp G, Cruz I: Mentoring college students: A critical review of the literature between 1990 and 2007. Res High Educ 2009, 50(6):525-545.

40. Blinn-Pike L: The benefits associated with youth mentoring relationships. In The Blackwell Handbook of Mentoring. Edited by Allen TD, Eby LT. Oxford: Wiley-Blackwell; 2011:355-395.

41. Grossman JB, Rhodes JE: The test of time: predictors and effects of duration in youth mentoring relationships. Am J Community Psychol 2002, 30(2):199-219.

42. Keller TE: Youth mentoring: theoretical and methodological issues. In The Blackwell Handbook of Mentoring: A Multiple Perspectives Approach. Edited by Allen TD, Eby LT. Oxford: Wiley-Blackwell; 2010:77-127.

43. Scrine C, Reibel T, Walker R: Key Findings of the Literature on Effective Mentoring Programs for Young People. Perth, Australia: Telethon Institute for Child Health Research; 2012.

44. Mellor S, Corrigan M: The Case for Change: a Review of Contemporary Research on Indigenous Education Outcomes. Camberwell, Victoria: Australian Council for Educational Research; 2004.
45. Rhodes JE: Stand by Me: The Risks and Rewards of Mentoring Today's Youth. Cambridge MA: Harvard University Press; 2002.

46. Minkler M, Wallerstein N: Improving health through community organization and community building: a health education perspective. In Community organizing and community building for health. Edited by Minkler M. New Brunswick, NJ: Rutgers University Press; 2005:26-50.

doi:10.1186/1471-2458-14-1263

Cite this article as: Bainbridge et al:: The quantity, quality and characteristics of Aboriginal and Torres Strait Islander Australian mentoring literature: a systematic review. BMC Public Health 2014 14:1263.

\section{Submit your next manuscript to BioMed Central and take full advantage of:}

- Convenient online submission

- Thorough peer review

- No space constraints or color figure charges

- Immediate publication on acceptance

- Inclusion in PubMed, CAS, Scopus and Google Scholar

- Research which is freely available for redistribution 